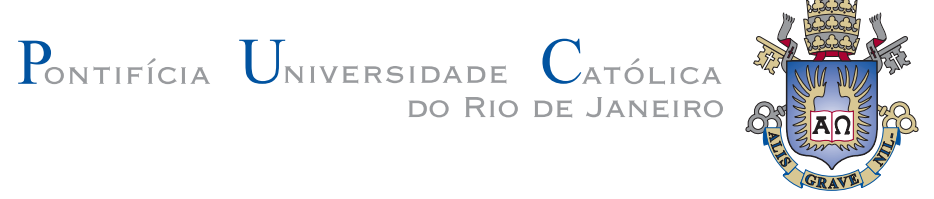

João Marcos Breia Jucá

\title{
A Capa de Invisibilidade como um Problema Inverso em Eletromagnetismo e Técnicas de Homogeneização
}

Dissertação de Mestrado

Dissertação apresentada ao Programa de Pós-graduação em Matemática da PUC-Rio como requisito parcial para obtenção do grau de Mestre em Matemática.

Orientador: Prof. Alex Lucio Ribeiro Castro 
João Marcos Breia Jucá

\begin{abstract}
A Capa de Invisibilidade como um Problema Inverso em Eletromagnetismo e Técnicas de

Homogeneização
\end{abstract}

Dissertação apresentada ao Programa de Pós-graduação em Matemática da PUC-Rio como requisito parcial para obtenção do grau de Mestre em Matemática. Aprovada pela Comissão Examinadora abaixo assinada.

\author{
Prof. Alex Lucio Ribeiro Castro \\ Orientador \\ Departamento de Matemática - PUC-Rio \\ Prof. Thomas Lewiner \\ Pontifícia Universidade Católica do Rio de Janeiro \\ Prof. Alejandro Cabrera \\ Universidade Federal do Rio de Janeiro
}

Prof. Jair Koiller

Instituto Nacional de Metrologia, Qualidade e Tecnologia

Prof. José Eugênio Leal

Coordenador do Centro Técnico Científico - PUC-Rio

Rio de Janeiro, 10 de março de 2015 
Todos os direitos reservados. É proibida a reprodução total ou parcial do trabalho sem autorização da universidade, do autor e do orientador.

\section{João Marcos Breia Jucá}

Bacharel em Matemática Pura - Universidade Federal Fluminense.

Ficha Catalográfica

Jucá, João Marcos Breia

A Capa de Invisibilidade como um Problema Inverso em Eletromagnetismo e Técnicas de Homogeneização / João Marcos Breia Jucá; orientador: Alex Lucio Ribeiro Castro. - Rio de Janeiro: PUC-Rio, Departamento de Matemática, 2015.

v., 69 f: il. ; $29,7 \mathrm{~cm}$

1. Dissertação (mestrado) - Pontifícia Universidade Católica do Rio de Janeiro, Departamento de Matemática.

Inclui referências bibliográficas.

1. Matemática - Dissertação. 2. Camuflagem. 3. Tomografia de Impedância Elétrica. 4. Metamaterial. 5. Equação de Laplace. 6. Problema Inverso. I. Castro, Alex Lucio Ribeiro. II. Pontifícia Universidade Católica do Rio de Janeiro. Departamento de Matemática. III. Título. 


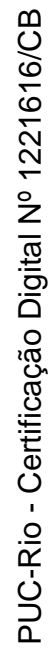

À minha mãe, Débora. 


\section{Agradecimentos}

Considerando as dificuldades em lograr êxito no âmbito profissional, principalmente nas regiões de extrema exclusão social, há que considerar os avanços na vida acadêmica um privilégio de poucos. Neste sentido, torna-se inevitável o registro de agradecimento:

A Deus, autor da minha vida. A Ele toda honra e glória;

Aos meus pais, Celso e Débora, pelo apoio ao longo de todos esses anos;

Ao meu amado irmão, Pedro Paulo, que sempre esteve ao meu lado ajudando e dando-me ânimo;

A minha querida avó, Adélia, pelas suas orações incessantes;

A minha amada Natália Cavalleiro, companheira de todas as horas, pelo carinho e ombro em momentos difíceis;

Aos demais familiares, por todo apoio;

Aos meus amigos, pelo companheirismo e apreço de sempre, em especial aos amigos Rafael Martins Lobosco, Vitor Hugo Pontes Ferreira e Isabella Paschuini, por toda ajuda dispensada e sugestões valiosas - grandes amigos;

Ao meu orientador, Alex Castro, por toda atenção dispensada;

Aos demais membros da banca, pela valiosa contribuição, em especial aos professores Thomas Lewiner e Jair Koiller;

A PUC-Rio, por toda estrutura e bolsa de isenção;

A CAPES, pelo incentivo à pesquisa, bolsa e taxa. 


\title{
Resumo
}

\author{
Jucá, João Marcos Breia; Castro, Alex Lucio Ribeiro. A Capa de \\ Invisibilidade como um Problema Inverso em Eletromag- \\ netismo e Técnicas de Homogeneização. Rio de Janeiro, 2015. \\ 69p. Dissertação de Mestrado - Departamento de Matemática, \\ Pontifícia Universidade Católica do Rio de Janeiro.
}

Invisibilidade sempre mexeu com a imaginação de crianças e adultos. Quem nunca imaginou ser capaz de tornar-se invisível em certas ocasiões? Recentemente essa ideia da ficção científica tomou forma na vida real, e um dos objetivos do presente texto é explicar de uma maneira acessível as principais ideias físicas e matemáticas por trás do conceito de invisibilidade. Pedimos do leitor somente uma modesta familiaridade com Cálculo Vetorial, Séries de Fourier e Álgebra Linear. O objetivo da capa de invisibilidade é tornar um objeto não detectável por meio de energia eletromagnética. A capa é fisicamente realizada por um metamaterial especialmente projetado para redirecionar certas ondas eletromagnéticas irradiadas sobre o objeto. Nesta exposição, usaremos como exemplo a tomografia de impedância elétrica (TIE) como método de deteç̧ão e explicaremos como criar uma capa invisível à TIE. Cabe ressaltar que o processamento de imagem através da TIE diz respeito a um problema inverso e, no contexto das equações diferenciais, esse problema envolve, a partir de determinadas simplificações, a equação de Laplace com condições de contorno. Despretensiosamente, optamos pelo caso bidimensional para facilitar a exposição das idéias principais, embora todos os nossos resultados possam ser generalizados em 3 dimensões.

\section{Palavras-chave}

Camuflagem; Tomografia de Impedância Elétrica; Metamaterial; Equação de Laplace; Problema Inverso; 


\section{Abstract}

Jucá, João Marcos Breia; Castro, Alex Lucio Ribeiro (advisor) . Invisibility Cloak as an Inverse Problem in Electromagnetism and Homogenization Techniques. Rio de Janeiro, 2015. 69p. M.Sc. Dissertation — Departamento de Matemática, Pontifícia Universidade Católica do Rio de Janeiro.

Invisibility has always instigated children and adult's imagination. Who never thought of occasionally being able to turn yourself invisible? Recently, this science fiction idea has taken shape in real life, and one of the objectives of this text is explain the main physical and mathematical ideas behind the invisibility concept, on a comprehensible way. We only require the reader has a modest familiarity with Vectorial Calculus, Fourier Series and Linear Algebra. Invisibility cloak aims to turn an object imperceptible to electromagnetic energy detection. The cloak is made of an especially projected metamaterial that redirects certain electromagnetic waves irradiated over the object. Here we will take as an example electrical impedance tomography (EIT) as a detection method and we will explain how to create an invisible cloak for EIT. It is worth mentioning that image processing through EIT is an inverse problem. Thereby, in the context of differential equations, this problem involves a few simplifications in the Laplace's problem with contours conditions. Unpretentiously, we chose the two-dimensional case to simplify the exposition of the main ideas, although all of our results may be generalized in three-dimensional case.

\section{Keywords}

Cloaking; Electrical Impedance Tomography; Metamaterial; Laplace's Equation; Inverse Problem; 


\section{Sumário}

1 Introdução 11

2 Preliminares $\quad 13$

2.1 Superposição 13

2.2 Condições de Contorno e Iniciais 16

2.3 O Método de Separação de Variáveis 19

3 O modelo Básico $\quad 22$

3.1 Condução elétrica 22

3.2 Condução Isotrópica 24

$\begin{array}{ll}3.3 \text { O caso Anisotrópico } & 26\end{array}$

$\begin{array}{lll}3.4 & \text { Tomografia de impedância } & 28\end{array}$

$\begin{array}{ll}3.5 & \text { Imageamento de regiões vazias }\end{array}$

3.6 Problemas inversos e camuflagem 31

3.7 Solução para equação de Laplace no anel 33

$\begin{array}{lll}3.8 & \text { Capa Inadequada } & 35\end{array}$

4 Construção da Camuflagem $\quad 39$

4.1 Mudança de Variáveis $\quad 39$

4.2 Criando a capa 42

4.3 A condutividade $\sigma$ é uma camuflagem aproximada 44

4.4 Comportamento na região de camuflagem 45

5 Conclusão $\quad 50$

$\begin{array}{ll}\text { Referências Bibliográficas } & 53\end{array}$

A Alguns resultados pertinentes $\quad \mathbf{5 5}$

A.1 Impedância elétrica e formas diferenciais das equações de Maxwell $\quad 55$

A.2 Teorema da Divergência e as Identidades de Green 56

A.3 Identidade de Parseval $\quad 65$

A.4 Um exemplo de interpolação $\quad 67$ 


\section{Lista de Figuras}

3.1 A região $\Omega$ esconde um objeto de um observador externo 23

3.2 Funcionamento tomografia de impedância elétrica

3.3 A imagem do lado esquerdo é uma imagem de impedância de uma seção transversal do tronco, feita com o coração preenchido com o sangue que saiu dos pulmões do indivíduo. A área do coração aparece em vermelho neste momento por consequência da alta condutividade (sangue é um bom condutor). Em contraste, nesse instante, os pulmões têm pouco sangue e são mostrados em azul. Na imagem à direita, o sangue já teria deixado o coração e preenchido os pulmões, invertendo as cores. (Figura extraída de [5]-Imagens cedidas por David Isaacson e do grupo de imagem por impedância elétrica do Instituto Politécnico Rensselaer, obtidas a partir de seu sistema de imagem de impedância ACT III)

3.4 Comparação de soluções no disco unitário e no anel. O gráfico à esquerda mostra linhas do fluxo de corrente $\mathbf{J}=-\gamma \nabla u$, onde $u$ é a solução para a equação de Laplace com condição de Dirichlet $f(\theta)=\cos (\theta)+\sin (\theta)$ (o potencial aplicado pelo observador) na fronteira do disco unitário. O fluxo mostrado no gráfico do meio tem o mesmo potencial aplicado $f$ na fronteira exterior, com a condição de Neumann nula do fluxo na fronteira interior do anel $1 / 2 \leqslant r \leqslant 1$. O gráfico mais à direita compara $\mathbf{J}$ na fronteira do disco e do anel, onde as setas mais curtas correspondem a $\mathbf{J}$ para o anel.

4.1 Um exemplo de uma função $\phi(\mathbf{x})=\frac{\psi(\|\mathbf{x}\|)}{\|\mathbf{x}\|} \mathbf{x}$, onde $\psi(r)$ é definida via (4-8). Note que $\phi$ leva o círculo de raio $r$ no círculo de raio $\psi(r) .44$

4.2 Comparação de soluções descamufladas e camufladas nos anéis. Os gráficos da esquerda e do meio mostram o fluxo das linhas de corrente $\mathbf{J}=-\gamma \nabla u$, onde $u$ é uma solução da equação de Laplace com condições de Dirichlet $f(\theta)=\cos (\theta)+\sin (\theta)$ (o potencial aplicado pelo observador) na fronteira exterior dos anéis com condutividade constante. O gráfico à direita mostra fluxos de linha da corrente $\mathbf{J}=-\gamma \nabla v$ para o círculo aproximadamente camuflado com condutividade anisotrópica $\sigma$ correspondente a $\rho=\frac{1}{10}$.

A.1 Orientação positiva de $\partial \Omega$.

A.2 O teorema de Green também é válido para domínios multiplamente conexos.

A.3 Se a parametrização $\alpha$ dá uma orientação positiva a $\partial \Omega, \widehat{n}(t)$ é a normal externa unitária no ponto $\alpha(t)$.

A.4 Sistema de coordenadas polares centrado em $\xi$.

A.5 A orientação positiva de $\partial \Omega_{r}$ coincide com a orientação positiva de $\partial \Omega$ e com a orientação negativa de $\partial B(\xi ; r)$. 
A tarefa não é tanto ver aquilo que ninguém viu, mas pensar o que ninguém ainda pensou sobre aquilo que todo mundo vê. Arthur Schopenhauer 


\section{Introdução}

Invisibilidade sempre foi tema presente em quadrinhos e fição científica. Personagens heróicos utilizavam super poderes de invisibilidade ou capas de invisibilidade para se proteger ou atacar seus inimigos sem que fossem percebidos. Quem nunca sonhou em ter o super poder da invisibilidade ou mesmo ter uma capa para tornar-se imperceptível? Mágicos e ilusionistas descobriram como utilizar espelhos em tamanho natural para curvar a luz, a fim de criar a ilusão de que coisas desaparecem. Por outro lado, câmeras também podem ser utilizadas para filmar o que está atrás de uma pessoal ou objeto e projetar a imagem, para que essa pessoa ou objeto pareça invisível de frente. Os cientistas, por sua vez, criaram metamateriais para guiar raios de luz ao redor de pequenos objetos bidimensionais. A propriedade essencial da capa não é simplesmente esconder a pessoa que está por baixo dela, um lençol seria suficiente para esse fim. Porém, tornando-se o portador invisível, a capa na verdade esconde o fato de que alguma coisa está sendo escondida, ocultada.

Na série de televisão "Star Trek", a pseudoexplicação para o funcionamento da camuflagem é decorrente de que "a curvatura seletiva dos raios de luz" ao redor do objeto a ser camuflado pode torná-lo invisível. Porém, com as leis da Física presentes no mundo real isso seria possível, mesmo que teoricamente? Recentemente, físicos e matemáticos descobriram que a resposta a essa questão é um qualificado "sim".

Na vida real, a ideia para se obter a camuflagem é projetar um "metamaterial" com micro estrutura especial capaz de dobrar ondas eletromagnéticas de maneira quantitativa e controlável. Cientistas e engenheiros já obtiveram progressos nesta direção e construíram metamateriais que ocultam objetos, em circunstâncias restritas, com sucesso.

Em 2006, John Pendry, David Schuring e David Smith publicaram uma ideia para uma capa, o que tornaria um objeto em uma ou mais dimensões invisível à sondagem por ondas eletromagnéticas em uma frequência fixa, rodeando-a com um metamaterial especialmente projetado. Logo depois, na universidade de Duke, o grupo de Smith construiu um dispositivo eficaz baseado em uma variante dessa ideia, e em janeiro de 2009 eles relataram 
a construção de um dispositivo que funciona para uma ampla gama de frequencias em duas dimensões.

Em princípio, as mesmas técnicas podem ser escaladas para trabalhar em comprimentos de ondas ópticas. Anteriormente, em 2003, Greenleaf, Lassas e Uhlmannjá haviam descrito essencialmente a mesma noção em um estudo sobre o problema inverso para tomografia de impedância elétrica empregada por Calderón. Mais recentemente, este grupo já descobriu um "duplo-revestimento" que pode encobrir fontes que iluminam ativamente (por exemplo, uma fonte de luz).

Este trabalho fornece uma explicação elementar, mas quantitativa, matematicamente honesto, da ideia essencial por trás da camuflagem, seguindo o método de mudança de variáveis descrito em [5], podendo ser considerado como uma versão anotada e adaptada deste mesmo artigo da SIAM review. 


\section{2 \\ Preliminares}

Neste capítulo abordaremos os conteúdos relevantes sobre o Método de Separação de Variáveis ou Método de Fourier. Apresentaremos, também, uma breve revisão de Álgebra Linear aplicada principalmente aos problemas que envolvem EDP's. Consideramos que o leitor tenha uma certa familiaridade com os principais conceitos e resultados referentes às séries de Fourier, Álgebra Linear básica e EDO's. Supomos, ainda, que o leitor está familiarizado com as propriedades elementares de funções $u: \Omega \subset \mathbb{R}^{n} \rightarrow \mathbb{R}$, em particular, com os conceitos de continuidade, diferenciabilidade e derivadas parciais. Se $u\left(x_{1}, x_{2}, \ldots, x_{n}\right)$ é uma função de várias variáveis, usaremos alguns tipos de notações para as derivadas parciais de $u$. Por exemplo, a derivada parcial de $u$ em relação a primeira variável $x_{1}$ poderá ser denotada por

$$
\frac{\partial u}{\partial x_{1}}, u_{x_{1}}, \partial_{x_{1}} u \text { ou } D_{1} u \text {. }
$$

Analogamente, as derivadas de segunda ordem poderão ser denotadas por

$$
\frac{\partial^{2} u}{\partial x_{1}^{2}}, u_{x_{1} x_{1}}, \partial_{x_{1}}^{2} u \text { ou } D_{1}^{2} u
$$

quando em relação à mesma variável $x_{1}$ e, no caso de variáveis diferentes, derivando primeiro em relação a $x_{1}$ e depois a $x_{2}$,

$$
\frac{\partial^{2} u}{\partial x_{2} \partial x_{1}}, u_{x_{1} x_{2}}, \partial_{x_{2}} \partial_{x_{1}} u \text { ou } D_{2} D_{1} u
$$

\section{1}

\section{Superposição}

As considerações a seguir são válidas para EDP's lineares de qualquer ordem, todavia, para fixarmos as ideias, vamos considerar uma EDP de primeira ou segunda ordem com $n$ variáveis independentes $x_{1}, x_{2}, \ldots, x_{n}$. Usaremos a notação vetorial $\mathbf{x}=\left(x_{1}, x_{2}, \ldots, x_{n}\right)$. Então, temos a seguinte equação:

$$
\sum_{i, j}^{n} a_{i j}(\mathbf{x}) D_{i} D_{j} u(\mathbf{x})+\sum_{j=1}^{n} b_{j}(\mathbf{x}) D_{j} u(\mathbf{x})+c(\mathbf{x}) u(\mathbf{x})+d(\mathbf{x})=0 .
$$


Note que se a ordem da EDP é 1 , então $a_{i j} \equiv 0$ quaisquer que sejam $1 \leq i, j \leq n$ e existe $j, 1 \leq j \leq n$, tal que $b_{j} \neq 0$.

Podemos reescrever a equação (2-1) na forma:

$$
L u=f
$$

onde $f(\mathbf{x})=-d(\mathbf{x})$ e

$$
(L u)(\mathbf{x})=\sum_{i, j}^{n} a_{i j}(\mathbf{x}) D_{i} D_{j} u(\mathbf{x})+\sum_{j=1}^{n} b_{j}(\mathbf{x}) D_{j} u(\mathbf{x})+c(\mathbf{x}) u(\mathbf{x})
$$

A cada função $u$ (suficientemente diferenciável) corresponde uma única função Lu; assim definimos um operador diferencial parcial L. Mais precisamente, seja $\Omega \subset \mathbb{R}^{n}$ aberto e suponha que as funções $a_{i j}, b_{j}$ e $c, 1 \leq i, j \leq n$, são contínuas em $\Omega$ e tomam valores reais, podemos então definir:

$$
\begin{aligned}
L: C^{k}(\Omega) & \rightarrow C(\Omega) \\
u & \mapsto L u
\end{aligned}
$$

onde $L u$ é dado pela fórmula $(2-3)$ e $C^{k}(\Omega)$ (respectivamente $\left.C(\Omega)\right)$ é o conjunto das funções $u: \Omega \rightarrow \mathbb{R} k$ vezes continuamente diferenciável (respectivamente contínua), com $k=1$ ou 2 .

Pelo fato de (2-1) ser linear, o operador $L$ em (2-3) é um operador linear, isto é, $L(0)=0$ e

$$
L(u+\alpha v)=L u+\alpha L v
$$

para quaisquer $u, v \in C^{k}(\Omega)$ e $\alpha \in \mathbb{R}$.

Podemos associar à EDP não homogênea (2-2) a EDP linear homogênea

$$
L u=0
$$

que é chamada a equação homogênea associada à equação (2-2). Por indução e linearidade de $L$, vemos que qualquer combinação linear de soluções da equação (2-5) é também solução, isto é, se $u_{1}, \ldots, u_{m}$ satisfazem $(2-5)$ e se $\alpha_{1}, \ldots, \alpha_{m}$ são escalares, então

$$
u=\sum_{j=1}^{m} \alpha_{j} u_{j}
$$

é também solução de (2-5).

Em linguagem de álgebra linear, $L$ é um operador linear definido em um espaço vetorial $V$ de funções $\left(V=C^{k}(\Omega)\right)$ e as soluções $u \in V$ da equação (2-5) formam um subespaço de $V$. Esse resultado é conhecido como o princípio da superposição (na sua forma finita). Entretanto, o espaço de soluções da equação (2-5) pode ter dimensão infinita. Em outras palavras, será que existe uma forma infinita do pincípio da superposição? A resposta é sim, sob certas condições. 
Proposição 2.1.1 (Princípio da Superposição) Seja L um operador diferencial parcial linear de ordem $k$ cujos coeficientes estão definidos em um aberto $\Omega \subset \mathbb{R}^{n}$. Suponha que $\left\{u_{m}\right\}_{m \in \mathbb{N}}$ é um conjunto de funções $C^{k}$ em $\Omega$ satisfazendo a EDP linear homogênea (2-5). Então, se $\left\{\alpha_{m}\right\}_{m \in \mathbb{N}}$ é uma sequência de escalares tal que a série:

$$
u(\mathbf{x})=\sum_{m=1}^{+\infty} \alpha_{m} u_{m}(\mathbf{x})
$$

é $k$ vezes diferenciável termo a termo em $\Omega$ e converge (uniformemente), $u$ satisfaz (2-5).

Prova: Enunciamos a proposição no caso geral mas faremos a demonstração no caso em que $k=1$ ou $k=2$, isto é, $L$ é definido por (2-3). Nesse caso, por hipótese, quaisquer que sejam $\mathbf{x} \in \Omega, 1 \leq i, j \leq n$,

$$
\begin{aligned}
u(\mathbf{x}) & =\sum_{m=1}^{+\infty} \alpha_{m} u_{m}(\mathbf{x}) \\
D_{i} u(\mathbf{x}) & =\sum_{m=1}^{+\infty} \alpha_{m} D_{i} u_{m}(\mathbf{x}) ; \\
D_{i} D_{j} u(\mathbf{x}) & =\sum_{m=1}^{+\infty} \alpha_{m} D_{i} D_{j} u_{m}(\mathbf{x})
\end{aligned}
$$

e essas séries convergem (uniformemente). Portanto, para todo $\mathrm{x} \in \Omega$,

$$
\begin{aligned}
(L u)(\mathbf{x}) & =\sum_{i, j=1}^{n} a_{i j}(\mathbf{x}) D_{i} D_{j} u(\mathbf{x})+\sum_{j=1}^{n} b_{j}(\mathbf{x}) D_{j} u(\mathbf{x})+c(\mathbf{x}) u(\mathbf{x}) \\
& =\sum_{m=1}^{+\infty} \alpha_{m}\left[\sum_{i, j=1}^{n} a_{i j}(\mathbf{x}) D_{i} D_{j} u_{m}(\mathbf{x})+\sum_{j=1}^{n} b_{j}(\mathbf{x}) D_{j} u_{m}(\mathbf{x})+c(\mathbf{x}) u_{m}(\mathbf{x})\right] \\
& =\sum_{m=1}^{+\infty} \alpha_{m}\left(L u_{m}\right)(\mathbf{x})=0
\end{aligned}
$$

o que demonstra a proposição no caso em que $k=1$ ou 2 .

$\mathrm{Na}$ seção seguinte daremos algumas definições para problemas que envolvem EDP's e apresentaremos alguns exemplos clássicos objetivando fixar ideias. 


\section{2}

\section{Condições de Contorno e Iniciais}

Uma grande diferença entre EDO's e EDP's é a informação suplementar necessária para unicidade de solução. Por exemplo, na solução geral de uma EDO linear aparecem uma ou mais constantes arbitrárias que podem ser determinadas quando impomos condições iniciais, isto é, fixando os valores da solução e de suas derivadas até certa ordem em um determinado ponto; também podemos obter unicidade, no caso de intervalos finitos, impondo condições nos extremos dos intervalos, as chamadas condições de contorno (como nos problemas de Sturm-Louville - que veremos mais adiante!). A situação para EDP's é fundamentalmente distinta. Mesmo no caso linear, a solução geral, quando é possível encontrá-la, envolve funções arbitrárias das variáveis independentes, de modo que existe um grau de generalidade muito maior com relação à forma da solução.

No caso de EDP's, cujo espaço de variáveis independentes é multidimensional, procuramos soluções definidas em um aberto $\Omega \subset \mathbb{R}^{n}$. Nesse contexto, torna-se natural substituir os extremos do intervalo (caso $n=1$ ) pela fronteira $\partial \Omega$ da região $\Omega$. Quando impomos condições sobre o valor da solução e de suas derivadas na fronteira da região (condiçôes de contorno) temos um problema de valores de contorno ou, simplesmente, problemas de contorno. Condições de contorno aparecem frequentemente na descrição de fenômenos físicos estacionários, isto é, independentes do tempo. Encontraremos muitas vezes condições do tipo

$$
\alpha u(x)+\beta \frac{\partial u}{\partial n}=f(x), x \in \partial \Omega
$$

onde $\alpha$ e $\beta$ são constantes dadas, $f$ é uma função dada em $\partial \Omega$ e $\frac{\partial u}{\partial n}$ é a derivada de $u$ na direção normal a $\partial \Omega$. No caso em que $\beta=0$, a condição (2-8) é conhecida como condições de Dirichlet; no caso em que $\alpha=0$, temos uma condição de Neumann.

Em problemas físicos dependentes do tempo, como é o caso de fenômenos de difusão e de fenômenos oscilatórios, é muitas vezes conveniente separar a variável temporal $t$ das demais variáveis espaciais $x, y, z$. O que ocorre muitas vezes é que os valores da solução e de suas derivadas em relação ao tempo até a ordem $k-1$ (supondo que a EDP é de ordem $k$ em $t$ ) são descritos no instante $t=0$ como função de $x, y, z$, assim, generalizamos o conceito de condições iniciais impondo o valor da solução e suas derivadas normais ao longo da curva $(n=2)$ ou superfície $(n=3)$ inicial $t=0$. O problema correspondente é um problema de Cauchy ou de valor inicial. Juntamente com as condições iniciais, podemos impor condições de contorno, para todo $t \geq 0$, em relação às 
variáveis espaciais: tais problemas são chamados de problemas mistos.

Vamos tentar fixar os conceitos acima com os exemplos a seguir.

Exemplo 2.2.1 Seja $\Omega \subset \mathbb{R}^{3}$ um aberto limitado ( $\Omega$ é o interior de um sólido $\bar{\Omega})$. Vamos denotar por $x=\left(x_{1}, x_{2}, x_{3}\right)$ os pontos de $\mathbb{R}^{3}$ e por $\Delta$ o operador laplaciano em $\mathbb{R}^{3}\left(\Delta u(x)=\frac{\partial^{2} u}{\partial x_{1}^{2}}+\frac{\partial^{2} u}{\partial x_{2}^{2}}+\frac{\partial^{2} u}{\partial x_{3}^{2}}\right)$. Então o problema

$$
\begin{aligned}
u_{t} & =\alpha^{2} \Delta u \text { em } \Omega \times(0,+\infty) \\
u(x, t) & =0, x \in \partial \Omega, t \geq 0 \\
u(x, 0) & =f(x), x \in \bar{\Omega}
\end{aligned}
$$

é um problema misto.

A condição $u(x, t)=0$ para $x \in \partial \Omega$ e $t \geq 0$ é uma condição de contorno, enquanto que a condição $u(x, 0)=f(x)$ para $x \in \bar{\Omega}$ é uma condição inicial. A função $f$ é dada, $\alpha^{2}$ é uma constante positiva e procuramos uma solução $u \in C(\bar{\Omega} \times[0,+\infty)) \cap C^{2}(\Omega \times(0,+\infty))$, logo $f$ tem que estar em $C(\bar{\Omega})$. Observe que, para que haja solução, $f$ tem que satisfazer uma condição de compatibilidade

$$
f(x)=0, \forall x \in \partial \Omega .
$$

Cabe observar que em problemas mistos, a condição de contorno e a condição inicial não são inteiramente independentes e é preciso então que seja satisfeita uma condição de compatibilidade para que haja solução. Fisicamente, o problema (2-9) descreve a temperatura $u(x, t)$ no ponto $x$ e no instante $t$ do sólido $\bar{\Omega}$ feito de material homogêneo, com difusividade térmica igual a $\alpha^{2} \mathrm{e}$ colocado em um reservatório térmico mantido à temperatura constante igual a zero (condições de contorno) com uma distribuição inicial de temperatura $f(x)$ (condição inicial). O problema análogo a uma dimensão espacial é

$$
\begin{aligned}
u_{t} & =\alpha^{2} u_{x x} \text { em }(0, l) \times(0,+\infty) \\
u(0, t) & =u(l, t)=0, t \geq 0 \\
u(x, 0) & =f(x), x \in[0, l]
\end{aligned}
$$

com condição de compatibilidade

$$
f(0)=f(l)=0 .
$$

No lugar do sólido $\bar{\Omega}$ temos agora uma barra de seção reta uniforme (com área muito pequena em relação ao comprimento) e comprimento $l$. Supomos, 
nesse caso, que não há troca de calor com o exterior através da superfície lateral da barra (os extremos correspondem à fronteira de $\Omega$ e estão mantidos à temperatura constante zero, pela condição de contorno).

Exemplo 2.2.2 O problema para a equação de onda em um intervalo finito

$$
\begin{aligned}
u_{t t} & =c^{2} u_{x x} \text { em }(0, l) \times(0,+\infty) \\
u(0, t) & =0=u(l, t), t \geq 0 \\
u(x, 0) & =f(x), x \in[0, l] \\
u_{t}(x, 0) & =g(x), x \in[0, l]
\end{aligned}
$$

pode também ser considerado como misto (condições iniciais $u(x, 0)=f(x)$, $u_{t}(x, 0)=g(x), x \in[0, l]$ e condições de contorno $\left.u(0, t)=0=u(l, t), t \geq 0\right)$. Note que, como a equação de onda é de segunda ordem em relação à variável temporal $t$, precisamos de duas condições iniciais. Para que exista solução é preciso que $f$ satisfaça a condição de compatibilidade

$$
f(0)=0=f(l) .
$$

No contexto físico, o problema (2-13) descreve uma corda elástica de comprimento $l$, presa nas pontas, vibrando em um plano vertical (uma corda de violão, por exemplo); $u(x, t)$ é o deslocamento vertical da corda no ponto $x$ no instante $t$ e as funções $f$ e $g$ descrevem, respectivamente, a posição e a velocidade iniciais da corda.

A generalização do problema (2-13) com duas variáveis espaciais fica

$$
\begin{aligned}
u_{t t} & =c^{2} \Delta u \text { em } \Omega \times(0,+\infty) \\
u\left(x_{1}, x_{2}, t\right) & =0,\left(x_{1}, x_{2}\right) \in \partial \Omega, t \geq 0 \\
u\left(x_{1}, x_{2}, 0\right) & =f\left(x_{1}, x_{2}\right),\left(x_{1}, x_{2}\right) \in \bar{\Omega} \\
u_{t}\left(x_{1}, x_{2}, 0\right) & =g\left(x_{1}, x_{2}\right),\left(x_{1}, x_{2}\right) \in \bar{\Omega}
\end{aligned}
$$

onde $\Omega \subset \mathbb{R}^{2}$ é aberto e limitado, e $\Delta$ é o operador laplaciano em $\mathbb{R}^{2}$. A condição de compatibilidade nesse caso é

$$
f\left(x_{1}, x_{2}\right)=0, \forall\left(x_{1}, x_{2}\right) \in \partial \Omega .
$$

O problema (2-15) descreve o movimento de uma membrana vibrando (como em um tambor). 
Exemplo 2.2.3 Seja $\Omega \subset \mathbb{R}^{2}$ um aberto. Então

$$
\begin{aligned}
\Delta u & =0 \mathrm{em} \Omega \\
u & =f \mathrm{em} \partial \Omega
\end{aligned}
$$

é um problema de contorno e de fato um problema de Dirichlet. A equação $\Delta u=0$ é denominada equação de Laplace. Em particular, estudaremos esse problema quando $\Omega$ for um disco.

Exemplo 2.2.4 Um outro problema de contorno para a equação de Laplace é

$$
\begin{aligned}
& \Delta u=0 \mathrm{em} \Omega \\
& \frac{\partial u}{\partial n}=f \mathrm{em} \partial \Omega
\end{aligned}
$$

que é conhecido por problema de Neumann.

Exemplo 2.2.5 Podemos, ainda, combinar os problemas (2-17) e (2-18):

$$
\begin{aligned}
\Delta u & =0 \mathrm{em} \Omega \\
u & =f \mathrm{em} \partial \Omega \\
\frac{\partial u}{\partial n} & =g \mathrm{em} \partial \Omega
\end{aligned}
$$

O problema (2-19) é denominado Problema de Dirichlet-Neumann. As condições de contorno $f$ e $g$ são chamadas dados de Dirichlet-Neumann.

Na próxima seção veremos que o Problema de Dirichlet-Neumann tem solução única (em uma determinada classe de funções) quando os dados de Dirichlet-Neumann atendem a determinadas condições. Apresentaremos alguns teoremas que revelarão em que sentido as condições impostas aos dados de Dirichlet-Neumann interferem na solução do problema (2-19).

\section{3}

\section{O Método de Separação de Variáveis}

O Método de Separação de Variáveis é um método que permite encontrar soluções de vários Problemas de Valor Inicial ou de Contorno em EDP's. Dado um determinado problema, a idéia básica do método é:

1. Separe a parte homogênea $(H)$ do seu problema, onde vale o Princípio da Superposição;

2. Procure autosoluções, isto é, soluções (não nulas) do sistema $(H)$; no caso do Método de Separação de Variáveis para duas variáveis,procuramos 
soluções do tipo $u(x, y)=X(x) Y(y)$. Sob uma expectativa otimista, encontraremos uma família de soluções - tipicamente $u_{1}, u_{2}, u_{3}, \ldots, u_{n}, \ldots$.

3. Pelo Princípio da Superposição, qualquer função do tipo $\sum_{n=1}^{m} c_{n} u_{n}(x, y)$ também será solução de $(H)$; Ainda usufruindo da expectativa otimista, procure uma solução do tipo $u(x, y)=\sum_{n=1}^{\infty} c_{n} u_{n}(x, y)$, ajustando os coeficientes $c_{i}$ de maneira a satisfazer as condições do problema inicial que não estavam em $(H)$. Espera-se, assim, encontrar uma solução do problema original.

Vamos usar o Método de Separação de Variáveis para resolver o seguinte problema:

Exemplo 2.3.1 Considere a equação de Laplace em coordenadas polares

$$
\begin{cases}u_{r r}+\frac{1}{r} u_{r}+\frac{1}{r^{2}} u_{\theta \theta}=0 & 0 \leq r<a \text { e } 0 \leq \theta \leq 2 \pi \\ u(a, \theta)=f(\theta) & 0 \leq \theta \leq 2 \pi\end{cases}
$$

em que $f$ é uma função periódica de período $2 \pi$ e a $>0$. Observe que o domínio (a região $\Omega$, como anteriormente), neste caso, é o interior do disco de raio $a>0$. Vamos procurar soluções do tipo $u(r, \theta)=R(r) \Theta(\theta)$. Se existem tais soluções, elas devem satisfazer:

$$
R^{\prime \prime} \Theta+\frac{1}{r} R^{\prime} \Theta+\frac{1}{r^{2}} R \Theta^{\prime \prime}=0
$$

multiplicando a equação (2-20) por $r^{2} \frac{1}{R \Theta}$ e separando $R$ e $\Theta$, temos:

$$
\frac{r^{2} R^{\prime \prime}+r R^{\prime}}{R}=-\frac{\Theta^{\prime \prime}}{\Theta}
$$

Como $r$ e $\theta$ são variáveis independentes, a equação (2-21) nos diz que ambos os membros dessa igualdade têm que ser constante, isto é,

$$
\frac{r^{2} R^{\prime \prime}+r R^{\prime}}{R}=-\frac{\Theta^{\prime \prime}}{\Theta}=\lambda
$$

Daí, extraímos duas EDO's:

$$
\begin{gathered}
\Theta^{\prime \prime}+\lambda \Theta=0 \\
\frac{d}{d r}\left(r R^{\prime}\right)=\frac{\lambda R}{r}
\end{gathered}
$$

Note que queremos $\Theta$ periódica de período $2 \pi$ já que, em coordenadas polares, $\theta=\alpha$ e $\theta=\alpha+2 \pi$ representam o mesmo ponto e, portanto, $u$ deve assumir $o$ 
mesmo valor para $\theta=\alpha$ e $\theta=\alpha+2 \pi$. Assim, o caso $\lambda<0$ não nos interessa (pois $\Theta$ seria uma combinação de funções exponenciais).

No caso $\lambda=0$ temos que $\Theta$ é uma função afim, o que só é periódica se for constante. Ai $\frac{d}{d r}\left(r R^{\prime}\right)=0 \Rightarrow r R^{\prime}=c_{1} \Rightarrow R=c_{1} \ln (r)+c_{2}$. Como $\ln (r) \rightarrow-\infty$ quando $r \rightarrow 0^{+}$, para que a solução seja finita, precisamos tomar $c_{1}=0$. Assim, a única solução encontrada para $\lambda=0$ é $u_{0}=$ cte.

Enfim, se $\lambda>0$ temos $\Theta(\theta)=c_{1} \cos (\sqrt{\lambda} \theta)+c_{2} \sin (\sqrt{\lambda} \theta)$. Como queremos $\Theta$ periódica de período $2 \pi$, precisamos tomar $\lambda=n^{2}$. Então temos $\Theta(\theta)=$ $c_{1} \cos (n \theta)+c_{2} \sin (n \theta)$ e $R(r)=c_{3} r^{n}+c_{4} r^{-n}$. Novamente, para que a solução seja finita quando $r \rightarrow 0^{+}$, precisamos tomar $c_{4}=0$. Assim, encontramos as seguintes soluções separadas:

$$
u_{n}(r, \theta)=r^{n}\left(A_{n} \cos (n \theta)+B_{n} \sin (n \theta)\right)
$$

Superpondo tudo, esperamos ter uma solução da forma

$$
u(r, \theta)=A_{0}+\sum_{n=1}^{\infty} r^{n}\left(A_{n} \cos (n \theta)+B_{n} \sin (n \theta)\right)
$$

Logo, tomando $r=a$, vem a condição

$$
u(a, \theta)=f(\theta)=A_{0}+\sum_{n=1}^{\infty} a^{n}\left(A_{n} \cos (n \theta)+B_{n} \sin (n \theta)\right)
$$

o que indica que esta é a série de Fourier de $f$. Em outras palavras, temos $A_{0}=\frac{a_{0}}{2} ; A_{n}=\frac{a_{n}}{a^{n}} ; B_{n}=\frac{b_{n}}{a^{n}}$, onde $f(\theta) \sim \frac{a_{0}}{2}+\sum_{n=1}^{\infty} a_{n} \cos (n \theta)+b_{n} \sin (n \theta)$.

É interessante escrever esta expressão usando a forma complexa. Ficaríamos ao invés com

$$
u(r, \theta)=\sum_{n=-\infty}^{\infty} c_{n}\left(\frac{r e^{i \theta}}{a}\right)^{n}
$$

onde $c_{n}=\frac{1}{2 \pi} \int_{0}^{2 \pi} f(\theta) e^{-i n \theta} d \theta$.

Esta abordagem indica apenas que encontramos um candidato a solução do problema. Precisaríamos, portanto, eleger esse candidato. Para isto, seria necessário garantir sob que condições o dado inicial $f$ será "bem comportado", isto é, possuirá série de Fourier e se esta série convirgirá, por exemplo, para o próprio $f$. Para informações sobre alguns resultados relevantes nesse contexto e sobre classes de funções aqui ditas apropriadas ou comportadas, veja apêndice. Para uma análise mais aprofundada em relação a existência e unicidade de soluções para os problemas aqui citados ou expostos, veja [7] e [6]. 


\section{3 \\ O modelo Básico}

\section{1}

\section{Condução elétrica}

O objetivo da camuflagem é tornar um objeto invisível, de modo que os observadores que olham na direção do objeto não conseguem vê-lo. As palavras "olhar" e "ver" aqui se referem aos observadores que usam ondas eletromagnéticas de alguma forma para descrever objetos. Por exemplo, o observador pode iluminar ativamente o objeto com radar, ou simplesmente fazer uso de ondas eletromagnéticas do ambiente, tais como a luz solar; não importa! Nesta seção, desenvolveremos um modelo matemático para uma técnica de elaboração de imagem conhecida como tomografia de impedância elétrica que faz com que seja fácil ilustrar a ideia através da camuflagem. Na próxima seção, mostraremos como disfarçar um objeto de modo que se torne quase ou completamente invisível para este tipo de imagem. No entanto, as técnicas aplicáveis às metodologias de imagem eletromagnéticas são muito gerais. No princípio, esses modelos encontraram aplicação em situações que pouco têm a ver com camuflagem ou eletromagnetismo, mas que geralmente ocorriam em fenômenos físicos ondulatórios; por exemplo, na modelagem de problemas acústicos (ondas sonoras) para implementação de programas computacionais que corrigem falhas ou ruídos de gravações musicais como também em modelos para aperfeiçoamento de dispositivos silenciosos no escapamento de veículos automotores; ondas mecânicas, como as ondas sísmicas produzidas por terremotos. Os modelos para este tipo de onda tornaram possível desde investigar o interior da Terra até projetar sistemas de alarme de terremotos que podem salvar milhares de vidas. Para problemas de ondas mecânicas, existem também modelos aplicáveis na construção de pontesque não quebram mesmo quando ocorrem oscilações provocadas pelas ondas do mar ou até mesmo do vento, como é o caso da ponte Rio-Niterói.

Podemos pensar no modelo para o processamento de imagem definindo as variáveis em $\mathbb{R}^{2}$ ou $\mathbb{R}^{3}$, mas para esta exposição será mais simples trabalharmos em um domínio limitado $\Omega$. Assumimos, apenas por conveniência, que $\Omega$ é 
o disco aberto unitário em $\mathbb{R}^{2}$ e usamos coordenadas retangulares $\left(x_{1}, x_{2}\right)$. Usamos $\partial \Omega$ para denotar a fronteira de $\Omega$, o círculo unitário. Suponhamos que um objeto esteja contido em $\Omega$ e um observador externo tenta obter a imagem deste objeto usando algum tipo de onda eletromagnética. Entretanto, o observador se limita a trabalhar apenas em $\partial \Omega$. O observador injeta ondas eletromagnáticas em $\Omega$, olha o que se exterioriza, e, em seguida, tenta deduzir a estrutura interior.

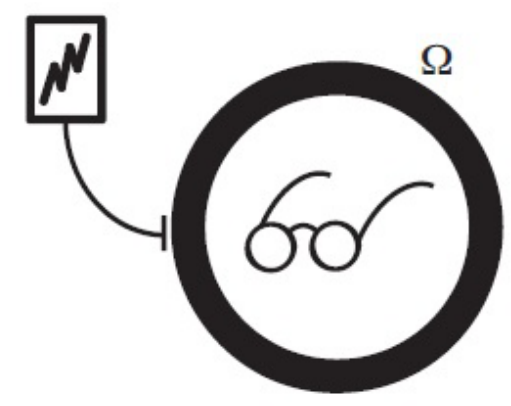

Figura 3.1: A região $\Omega$ esconde um objeto de um observador externo

Em geral, utilizam-se equações de Maxwell para quantificar o comportamento de campos eletromagnéticos, mas isto, em princípio, é desnecessário para o problema. Alguma simplificação pode ser obtida através da modelagem da situação com a equação da onda $\left(u_{t t}=c^{2} \Delta u\right)$.

Por exemplo, as componentes dos campos elétrico e magnético no espaço vazio obedecem a equação da onda. Podemos simplificar ainda mais quando consideramos apenas estado estacionário, ou seja, quando todas as quantidades são independentes do tempo. Além disso, o interior de $\Omega$ (mesmo quando "vazio") não consistirá de um espaço vazio, mas sim de um material eletricamente condutivo. Como se descreve em mais detalhes a seguir, o observador usará correntes e tensões elétricas previamente mensuradas para processar a imagem do interior de $\Omega$.

Comecemos por quantificar o que queremos dizer quando mencionamos que o interior de $\Omega$ é "vazio". Inicialmente, definiremos alguns conceitos no contexto físico: um material é considerado homogêneo se as suas propriedades físicas são as mesmas em todos os pontos e é dito isotrópico se o material não tem propriedades direcionais. Por exemplo, um bloco de madeira pode ser (aproximadamente) homogêneo, mas não é isotrópico, visto que a orientação da fibra apresenta comportamento físico direção-dependente. Dizemos ainda que um material que não é isotrópico é anisotrópico.

Assim, diremos que a região $\Omega$ está vazia se o interior de $\Omega$ é preenchido com um material eletricamente condutor que é homogêneo e isotrópico em 
relação à condução elétrica; Suponhamos que esta é a condição em que o observador externo espera encontrar $\Omega$. Evidentemente, se colocarmos um objeto dentro de $\Omega$, o objeto pode não ter as mesmas propriedades elétricas e vai alterar a forma como os fluxos de corrente elétrica seguem dentro de $\Omega$. Esta alteração pode ser usada para a detecção e elaboração da imagem do objeto do lado de fora de $\Omega$.

\section{2}

\section{Condução Isotrópica}

Para quantificar tudo isso, denotemos por $u\left(x_{1}, x_{2}\right)$ o potencial elétrico (a "voltagem") no ponto $\left(x_{1}, x_{2}\right) \in \Omega$.O campo elétrico $\mathbf{E}$ (um campo de vetores) em $\Omega$ satisfaz $\mathbf{E}=-\nabla u$. O campo elétrico puxa elétrons livres e impulsiona o fluxo de corrente, porém, usaremos o modelo "clássico de corrente", em que um fluxo de cargas positivas flui. Denotaremos por $\mathbf{J}$ o campo vetorial em $\Omega$ que descreve o fluxo de corrente. O modelo mais simples de como $\mathbf{J}$ depende de $\mathbf{E}$ e, portanto, de $u$, é:

$$
\mathbf{J}=\gamma \mathbf{E}
$$

onde $\gamma$ é a condutividade, isto é, a propriedade elétrica que mede a facilidade que um determinado material oferece à passagem de corrente elétrica quando submetido a uma diferença de potencial. No caso de um material isotrópico homogêneo, $\gamma$ é simplesmente uma constante não negativa, porém, mais geralmente, $\gamma$ pode ser uma função de posição de $\left(x_{1}, x_{2}\right)$, ou, no caso anisotrópico, uma matriz; ver seção 3.3 abaixo. A equação (3-1) é, em certo sentido, apenas uma versão bidimensional da lei de Ohm e postula uma relação linear entre o campo elétrico e o fluxo de corrente, com a corrente fluindo sempre na direção de E. Se $\gamma$ torna-se cada vez maior $(\gamma \rightarrow+\infty)$, então um excesso de corrente flui para uma determinada intensidade do campo elétrico, ao passo que se $\gamma$ aproxima-se de zero $(\gamma \rightarrow 0)$, a corrente flui pouco. O caso extremo, $\gamma=0$, corresponde a um perfeito isolante -não importa quão forte o campo elétrico seja, não haverá corrente. De $\mathbf{E}=-\nabla u$ e (3-1) obtemos

$$
\mathbf{J}=-\gamma \nabla u
$$

Se a carga elétrica é conservada em $\Omega$, como deve ser, se não há fontes de corrente no interior, temos $\nabla \cdot \mathbf{J}=0$ em todo o interior de $\Omega$. Com (3-2), 
isso implica

$$
\nabla(\gamma \nabla u)=0, \quad \text { em } \Omega
$$

Há que se dar especial atenção ao caso em que $\gamma$ é constante. Nesse caso, com $\gamma$ constante (isto é, quando $\Omega$ está vazio), podemos simplificar (3-3) pela equação de Laplace:

$$
\Delta u=0, \quad \text { em } \Omega,
$$

onde $u=u\left(x_{1}, x_{2}\right)$. Esta é a equação diferencial parcial que deve ser satisfeita pelo potencial elétrico $u$ dentro de um condutor homogêneo isotrópico.

Cabe observar que as funções que satisfazem (3-4) são referidas como funções harmônicas. Evidentemente, qualquer função constante $u$ satisfaz (3-4) (que a partir de (3-2) corresponde a corrente zero em todos os pontos em $\Omega$ ), porém, o caso mais interessante ocorre quando a corrente é diferente de zero, e isso requer um potencial não constante em $\Omega$.

Como podemos obter um potencial não constante dentro de $\Omega$ ? Induzindo um potencial $f$ não constante sobre $\partial \Omega$, por exemplo, fixando eletrodos à $\partial \Omega$, de modo que

$$
u=f, \quad \text { em } \partial \Omega,
$$

para alguma função $f$ escolhida. Ou seja, a equação (3-5) é uma condição de fronteira, e $f$ é o dado de Dirichlet.

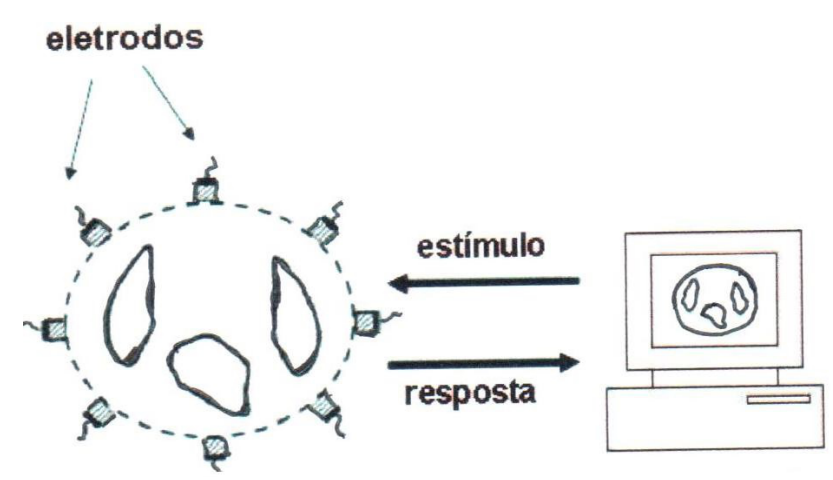

Figura 3.2: Funcionamento tomografia de impedância elétrica

Vejamos, então, um exemplo cuja solução é um potencial não constante.

Exemplo 3.2.1 Suponhamos que parametrizássemos a fronteira do disco da maneira usual,com $x_{1}=\cos \theta, x_{2}=\sin \theta, 0 \leq \theta<2 \pi$. Seja $f(\theta)=$ $a \cos \theta+b \sin \theta+c$, com $a, b, c$ constantes, os dados de Dirichlet no ponto 
correspondente em $\partial \Omega$. Nestas condições, a solução de (3-4) - (3-5) é a função harmônica $u\left(x_{1}, x_{2}\right)=a x_{1}+b x_{2}+c$

De fato, temos que $\Delta u\left(x_{1}, x_{2}\right)=0$, para $\left(x_{1}, x_{2}\right)=(0,0) \in \Omega$. Para $\left(x_{1}, x_{2}\right) \neq(0,0)$, escrevamos $x_{1}=r \cos \theta$ e $x_{2}=r \sin \theta$, onde $0<r \leq 1$ e $0 \leq \theta<2 \pi$. Assim, $u\left(x_{1}(r, \theta), x_{2}(r, \theta)\right)=$ ar $\cos \theta+b r \sin \theta+c$. Usando regra da cadeia e derivando em relação as variáveis $r$ e $\theta$, temos que $\Delta u(r, \theta)=$ $\frac{\partial^{2} u}{\partial r^{2}}+\frac{1}{r} \frac{\partial u}{\partial r}+\frac{1}{r^{2}} \frac{\partial^{2} u}{\partial \theta^{2}}=0+\frac{1}{r}(a \cos \theta+b \sin \theta)+\frac{1}{r^{2}}(-a r \cos \theta-b r \sin \theta)=0$. Além disso, quando $r=1$, temos $u(1, \theta)=a \cos \theta+b \sin \theta+c=f(\theta)$. Logo, concluimos que o problema equivalente: $\Delta u(r, \theta)=0$ para $(r, \theta) \in[0,1) \times[0,2 \pi)$ e $u(1, \theta)=f$, tem por solução $u(r, \theta)=\operatorname{ar} \cos \theta+b r \sin \theta+c$, satisfazendo, portanto (3-4) - (3-5). Observe que esta solução é a superposição das soluções constante e $n=1$ do exemplo 2.3.1.

A equação de Laplace (3-4) e a condição de fronteira de Dirichlet (3-5), em conjunto, constituem um problema de Dirichlet, com uma solução única $u$ para qualquer potencial aplicado $f$ que seja viável (por exemplo, contínuo). Ainda não encontramos a presença de um objeto dentro de $\Omega$, então (3-4) é apropriado apenas para um recipiente vazio em $\Omega$. Em uma seção posterior, mostraremos como modelar e detectar a presença de um "buraco" não condutor dentro de $\Omega$.

\section{3}

\section{O caso Anisotrópico}

Muitos materiais apresentam propriedades físicas anisotrópicas. No contexto da condução elétrica, isto significa que, em qualquer ponto, um material pode conduzir melhor em algumas direções do que em outras, de modo que o modelo de condução de (3-1) com $\gamma$ sendo um escalar é inadequado. Uma generalização natural de (3-1) é assumir que, em qualquer ponto do material, há uma direção de máxima condutividade e uma direção de condutividade mínima. Suponhamos que o material tenha máxima condutividade $\gamma_{M}>0$ na direção do vetor unitário $v_{M}$ e condutividade mínima $\gamma_{m}>0$ na direção do vetor unitário $v_{m}$, então $0<\gamma_{m} \leq \gamma_{M}$. Também é natural supor que os vetores de direção, $v_{M}$ e $v_{m}$, são ortogonais entre si. Um modelo que expõe esse comportamento é

$$
\mathbf{J}=\sigma \mathbf{E},
$$

onde $\sigma$ é uma matriz simétrica positiva definida $2 \times 2$ ( $\sigma$ pode depender da posição), uma vez que as matrizes positivas definidas simétricas têm autovetores ortogonais e autovalores positivos. (Lembre-se que uma matriz 
$A$ é positiva definida se $v^{t} A v>0$ para todos os vetores $v$ não nulos, onde $v^{t}$ é o transposto de $v$ ). O inverso também é verdadeiro: uma matriz com uma base ortogonal de autovetores e autovalores positivos é uma matriz simétrica positiva definida. Vejamos alguns exemplos:

Exemplo 3.3.1 Uma matriz $2 \times 2 \sigma$ que modela um condutor isotrópico com condutividade (escalar) $\gamma$ em todas as direções é:

$$
\sigma=\left(\begin{array}{ll}
\gamma & 0 \\
0 & \gamma
\end{array}\right)
$$

Exemplo 3.3.2 Uma matriz de condutividade anisotrópica $\sigma$ que modela um material homogêneo com condutividade geral $\gamma_{M}$ na direção do vetor unitário $v_{M}=\frac{\sqrt{2}}{2} \hat{i}+\frac{\sqrt{2}}{2} \widehat{j}$ e condutividade $\gamma_{m}$ na direção do vetor unitário $v_{m}=$ $\frac{\sqrt{2}}{2} \hat{i}-\frac{\sqrt{2}}{2} \widehat{j}$ é dada por:

$$
\sigma=\left(\begin{array}{ll}
\frac{\left(\gamma_{M}+\gamma_{m}\right)}{2} & \frac{\left(\gamma_{M}-\gamma_{m}\right)}{2} \\
\frac{\left(\gamma_{M}-\gamma_{m}\right)}{2} & \frac{\left(\gamma_{M}+\gamma_{m}\right)}{2}
\end{array}\right)
$$

Note que $\sigma$ é diagonalizável, isto é, $\sigma=P D P^{-1}=P D P^{t}$, onde $P$ é a matriz cujas colunas são autovetores de $\sigma$ e $D$ é a matriz diagonal de autovalores (na ordem das colunas de P).

Para condução anisotrópica, (3-6) substitui (3-1), e (3-3) torna-se

$$
\nabla(\sigma \nabla u)=0, \quad \text { em } \Omega
$$

Se um campo elétrico $\mathbf{E}$ é aplicado numa direção que é paralela à $v_{M}$, então o fluxo de corrente resultante é $\mathbf{J}=\sigma \mathbf{E}=\gamma_{M} \mathbf{E}$ tal que $\|\mathbf{J}\|=\gamma_{M}\|\mathbf{E}\|$. Para um valor fixado de $\|\mathbf{E}\|$, esta direção para $\mathbf{E}$ (paralela à $v_{M}$ ) maximiza $\|\mathbf{J}\|$; veja a proposição 3.3.3 abaixo. Da mesma forma tendo $\mathbf{E}$ paralelo a $v_{m}$ minimiza $\|\mathbf{J}\|$.

Proposição 3.3.3 Se $\sigma$ é uma matriz $n \times n$ positiva definida e fixamos $\|v\|=1$ então $\|\sigma v\|^{2}$ é maximizada quando $v$ é um autovetor para $\sigma$ correspondente ao maior autovalor para $\sigma$.

Prova: Inicialmente, como $\sigma$ é positiva definida, temos uma base de autovetores. Assim, seja $\left\{v_{1}, v_{2}, \ldots, v_{n}\right\}$ base de autovetores de $\sigma$ associada aos 
autovalores $0<\lambda_{1} \leq \lambda_{2} \leq \ldots \leq \lambda_{n}$. Seja $v=\sum_{k=1}^{n} \alpha_{k} v_{k}$. Temos:

$$
\begin{aligned}
\|\sigma v\|^{2} & =<\sigma v, \sigma v> \\
& =<\sum_{k=1}^{n} \alpha_{k} \lambda_{k} v_{k}, \sum_{k=1}^{n} \alpha_{k} \lambda_{k} v_{k}> \\
& =\sum_{k=1}^{n} \alpha_{k}{ }^{2} \lambda_{k}^{2}
\end{aligned}
$$

pois os $v_{k}$ são ortonormais. Nosso problema é, portanto, maximizar $\sum_{k=1}^{n} \alpha_{k}{ }^{2} \lambda_{k}{ }^{2}$ sujeito à $\sum_{k=1}^{n} \alpha_{k}^{2}=1$.

Agora, note que

$$
\begin{aligned}
\sum_{k=1}^{n} \alpha_{k}{ }^{2} \lambda_{1}{ }^{2} & \leq \sum_{k=1}^{n} \alpha_{k}{ }^{2} \lambda_{k}{ }^{2} \leq \sum_{k=1}^{n} \alpha_{k}{ }^{2} \lambda_{n}{ }^{2} \\
0 & <\lambda_{1}^{2} \leq \sum_{k=1}^{n} \alpha_{k}{ }^{2} \lambda_{k}{ }^{2} \leq \lambda_{n}{ }^{2} .
\end{aligned}
$$

E isto prova o resultado.

\section{4}

\section{Tomografia de impedância}

Na tomografia de impedância tenta-se imaginar o interior de $\Omega$ através da aplicação de uma corrente elétrica para $\partial \Omega$, digamos, anexando vários eletrodos em $\partial \Omega$. A corrente aplicada na fronteira induz uma variação espacial potencial (isto é, tensão) ao longo do interior de $\Omega$, induzindo o fluxo de corrente pelo interior. A corrente deve entrar ou sair de $\Omega$ através dos eletrodos conectados, e o potencial resultante sobre $\partial \Omega$ (que pode ser medido) depende das propriedades do interior de $\Omega$. A partir deste tipo de informação corrente aplicada e tensão resultante - pode-se deduzir informações sobre as propriedades elétricas do interior de $\Omega$, tais como a condutividade, e, assim, formar imagens. Veja figura 3.3 de um exemplo de uma imagem do coração e dos pulmões obtidos a partir de um sistema de imagem real da impedância.

O leitor interessado pode encontrar no apêndice uma dedução simplificada para o problema da tomografia de impedância elétrica. Neste caso, deduzimos a EDP que rege o potencial elétrico $u$ no interior de $\Omega$ a partir da formulação matemática da impedância elétrica e das formas diferenciais das equações de Maxwell. 

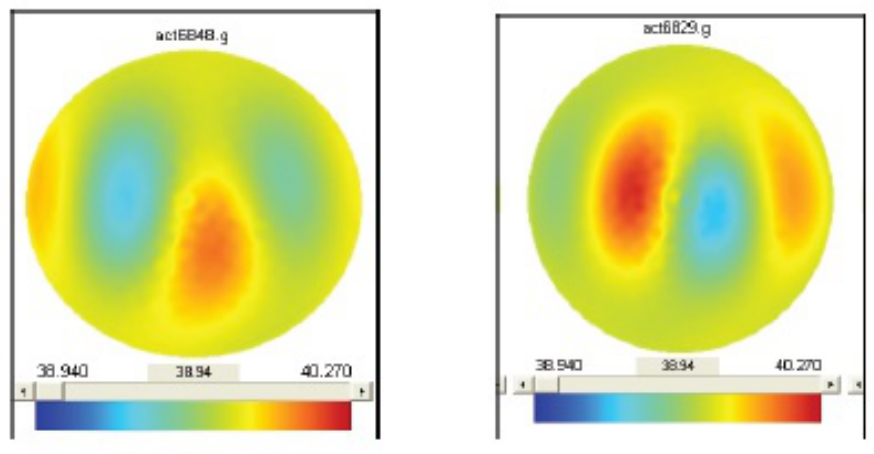

Figura 3.3: A imagem do lado esquerdo é uma imagem de impedância de uma seção transversal do tronco, feita com o coração preenchido com o sangue que saiu dos pulmões do indivíduo. A área do coração aparece em vermelho neste momento por consequência da alta condutividade (sangue é um bom condutor). Em contraste, nesse instante, os pulmões têm pouco sangue e são mostrados em azul. Na imagem à direita, o sangue já teria deixado o coração e preenchido os pulmões, invertendo as cores. (Figura extraída de [5]-Imagens cedidas por David Isaacson e do grupo de imagem por impedância elétrica do Instituto Politécnico Rensselaer, obtidas a partir de seu sistema de imagem de impedância ACT III)

\section{5}

\section{Imageamento de regiões vazias}

Vejamos como alguém pode imaginar alguns tipos especiais de objetos em $\Omega$ através da tomografia de impedância elétrica, injetando uma corrente e medindo o potencial resultante. Tomamos a abordagem equivalente, porém, mais matematicamente conveniente de aplicação de um potencial e, em seguida, medimos a corrente resultante em $\partial \Omega$. A chave é a de determinar a relação entre o potencial aplicado e a corrente resultante na fronteira de um objeto e como essa função depende das propriedades do interior de $\Omega$.

Para começar, suponha que $\Omega$ está vazio, isto é, tem condutividade isotrópica homogênea $\gamma>0$. Suponha que se coloque um objeto não condutor $D$ dentro de $\Omega$; pense em $D$ como um vácuo, isto é, ausente de matéria. Quando um potencial $f$ é aplicado a $\partial \Omega$, a presença do vácuo interrompe o fluxo de corrente no interior de $\Omega$, e este efeito deve ser observado a partir da fronteira. A quantidade observada é a velocidade com que a corrente flui em $\Omega$ em cada ponto em $\partial \Omega$. A velocidade à qual a corrente flui para fora, próximo a um ponto $p \in \partial \Omega$, é $\mathbf{J}(p) \cdot n(p)$, onde $n(p)$ é um vetor normal unitário que aponta para fora de $\partial \Omega$ no ponto $p$. Iremos, doravante, suprimir a dependência de quantidades como $\nabla u$ ou $n$ em $p$. Aplicação de (3-2) mostra que a corrente que flui para fora através de $\partial \Omega$ em um determinado ponto é $-\gamma \nabla u \cdot n$. A 
taxa em que a corrente entra por $\partial \Omega$ é $\gamma \nabla u \cdot n$, que é o dado de Neumann para a função $u$. A presença de $D$ em $\Omega$ altera o fluxo de corrente, para que nenhuma corrente possa fluir para dentro de $D$ a partir de $\Omega \backslash D$. Isso significa que $\mathbf{J} \cdot n=0$ em $\partial D$, onde aqui $n$ denota um vetor normal unitário em $\partial D$, digamos, apontando para dentro de $D$ (fora de $\Omega \backslash D$ ). A partir da equação (3-2) obtemos $\gamma \nabla u \cdot n=0$ em $\partial D$. Neste caso, o potencial $u$ é definido apenas em $\Omega \backslash D$ e obedece a equação de Laplace lá, junto com a condição de fronteira de Dirichlet (3-5) em $\partial \Omega$ e a condição de fronteira adicional

$$
\gamma \nabla u \cdot n=0, \quad \text { em } \partial D
$$

Como $\gamma>0$, também podemos escrever (3-8) simplesmente como $\frac{\partial u}{\partial n}=0$, usando a notação abreviada $\frac{\partial u}{\partial n}:=\nabla u \cdot n$ para a derivada normal.

Exemplo 3.5.1 Suponhamos que o observador aplique o potencial $f(\theta)=$ $\cos \theta+\sin \theta$ para a fronteira do disco. Do exemplo 3.2.1, o potencial resultante no interior do disco vazio é $u\left(x_{1}, x_{2}\right)=x_{1}+x_{2}$. Mas se tirarmos uma bola $D=B_{1 / 2}(0)$ (onde usamos $B_{r}(p)$ para denotar uma bola de raio $r$ centrada no ponto $p$, e 0 indica a origem), então $u\left(x_{1}, x_{2}\right)=x_{1}+x_{2}$ já não é o potencial induzido no anel $\Omega \backslash D$ pelo potencial $f$, porque u não satisfaz (3-8). Para ver isto, note que $\partial D$ pode ser parametrizada como $x_{1}=\frac{1}{2} \cos \theta, x_{2}=\frac{1}{2} \sin \theta$ $\operatorname{com} n=-\cos \theta \widehat{i}-\sin \theta \widehat{j}$. Então $\nabla u \cdot n=(\widehat{i}+\widehat{j})(-\cos \theta \widehat{i}-\sin \theta \widehat{j})=$ $-(\cos \theta+\sin \theta)$ o que não é identicamente nula em $\partial D$. Na verdade, neste caso, o potencial correto de dentro de $\Omega \backslash D$ é $u\left(x_{1}, x_{2}\right)=\frac{\left(x_{1}+x_{2}\right)\left(4 x_{1}^{2}+4 x_{2}^{2}+1\right)}{5 x_{1}^{2}+5 x_{2}{ }^{2}}$. Veja figura 3.4 para gráficos do fluxo de corrente J para cada caso. O vácuo não condutor impede o fluxo de corrente, e o observador mede (em coordenadas polares $(r, \theta)) \frac{\partial u}{\partial n}(1, \theta)=\frac{3}{5}(\cos \theta+\sin \theta)$ em $\partial \Omega$, em comparação a $\frac{\partial u}{\partial n}(1, \theta)=$ $\cos \theta+\sin \theta$ para o disco unitário intacto.

Podemos verificar qua a função $u\left(x_{1}, x_{2}\right)=\frac{\left(x_{1}+x_{2}\right)\left(4 x_{1}^{2}+4 x_{2}^{2}+1\right)}{5 x_{1}^{2}+5 x_{2}^{2}}$ do exemplo 3.5.1 é realmente harmônica em $\Omega \backslash D$ (onde $D=B_{1 / 2}(0)$ ) com $u=\cos \theta+\sin \theta$ em $\partial \Omega$ e $\nabla u \cdot n=0$ em $\partial D$.

Inicialmente, façamos $x_{1}=r \cos \theta, x_{2}=r \sin \theta$, para $\frac{1}{2} \leq r<1 \mathrm{e}$ $0 \leq \theta<2 \pi$. Vamos verificar que:

$$
\begin{aligned}
u_{r r}+\frac{1}{r} u_{r}+\frac{1}{r^{2}} u_{\theta \theta} & =0 \\
u(1, \theta) & =\cos \theta+\sin \theta \quad \text { em } \quad \partial \Omega \\
\nabla u \cdot n & =0 \quad \text { em } \quad \partial B_{\frac{1}{2}}(0) .
\end{aligned}
$$


Com efeito, $u(r, \theta)=\left(\frac{4}{5} r+\frac{1}{5 r}\right)(\cos \theta+\sin \theta)$ e, então, $u_{r}=\left(\frac{4}{5}-\frac{1}{5 r^{2}}\right)(\cos \theta+$ $\sin \theta), u_{r r}=\left(\frac{2}{5} \frac{1}{r^{3}}\right)(\cos \theta+\sin \theta), u_{\theta}=\left(\frac{4}{5} r+\frac{1}{5 r}\right)(-\sin \theta+\cos \theta), u_{\theta \theta}=$ $\left(\frac{4}{5} r+\frac{1}{5 r}\right)(-\cos \theta-\sin \theta)$. Assim,

$$
\begin{aligned}
u_{r r}+\frac{1}{r} u_{r}+\frac{1}{r^{2}} u_{\theta \theta} & = \\
& =\left(\frac{2}{5 r^{3}}+\frac{4}{5 r}-\frac{1}{r^{3}}-\frac{4}{5 r}-\frac{1}{5 r^{3}}\right)(\cos \theta+\sin \theta)=0
\end{aligned}
$$

Além disso, $\nabla u=\left(\frac{4 r^{2}-1}{5 r^{2}}\right)(\cos \theta+\sin \theta) \widehat{i}+\left(\frac{4 r^{2}+1}{5 r}\right)(\cos \theta-\sin \theta) \widehat{j} \mathrm{e}$ $n=-\frac{1}{2} \cos \theta \widehat{i}-\frac{1}{2} \sin \theta \widehat{j}$.
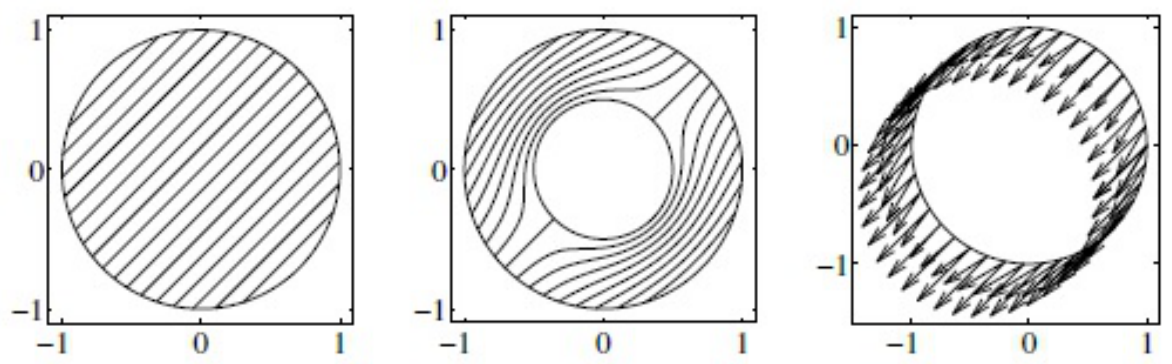

Figura 3.4: Comparação de soluções no disco unitário e no anel. O gráfico à esquerda mostra linhas do fluxo de corrente $\mathbf{J}=-\gamma \nabla u$, onde $u$ é a solução para a equação de Laplace com condição de Dirichlet $f(\theta)=\cos (\theta)+\sin (\theta)$ (o potencial aplicado pelo observador) na fronteira do disco unitário. O fluxo mostrado no gráfico do meio tem o mesmo potencial aplicado $f$ na fronteira exterior, com a condição de Neumann nula do fluxo na fronteira interior do anel $1 / 2 \leqslant r \leqslant 1$. O gráfico mais à direita compara $\mathbf{J}$ na fronteira do disco e do anel, onde as setas mais curtas correspondem a $\mathbf{J}$ para o anel.

\section{6}

\section{Problemas inversos e camuflagem}

A discussão acima sugere um processamento de imagem por impedância para a coleta de informações sobre o interior de $\Omega$, neste caso, encontrar um buraco em $\Omega$ :

1. Aplique um potencial $f$ para $\partial \Omega$ (equação 3-5);

2. Meça a resposta $\gamma \nabla u \cdot n$ em $\partial \Omega$ (medir a corrente resultante).

A partir deste tipo de "estímulo-resposta" ou dados de Dirichlet-Neumann desejamos determinar o tamanho exato, a forma e a localização do buraco $D$. 
Evidentemente os passos 1 e 2 podem ser repetidos com diferentes potenciais de entrada $f$, que podem produzir informações suplementares. Imagem de impedância é um exemplo de problema inverso. A definição de um problema inverso não é imutável, mas pode ser definido mais ou menos como um problema que propõe "deduzir a causa do efeito". No contexto das equações diferenciais, essa proposta toma frequentemente a forma de deduzir os coeficientes de uma equação diferencial a partir do conhecimento das soluções, ao invés do mais tradicional problema "direto" de encontrar a solução para uma equação diferencial específica com coeficientes conhecidos. Nesse caso, o problema inverso é deduzir qual região interior $D$ poderia ter rendido a atual fronteira medida para o potencial $f$ aplicado (em vez de serem dados $D$ e $f$, e pedir para calcular a atual fronteira resolvendo a equação diferencial). Problemas inversos desta forma muitas vezes ocorrem em aplicações onde se quer deduzir a estrutura interior a partir de medições exteriores.

Vamos retratar a definição de problema inverso no contexto da tomografia de impedância elétrica. Consideremos então as equações:

$$
\begin{array}{rrrr}
\nabla \cdot(-\sigma \nabla u)=0 & \text { em } & \Omega \\
u=f & \text { em } & \partial \Omega \\
-\sigma \frac{\partial u}{\partial n}=\overline{\mathbf{J}} & \text { em } & \partial \Omega
\end{array}
$$

em que o dado de Dirichlet $f$ é o potencial elétrico em $\partial \Omega$ (estímulo) e o dado de Neumann $\overline{\mathbf{J}}$ é a componente normal ao contorno do fluxo de corrente elétrica na fronteira $\partial \Omega$ (resposta).

Primeiramente, o problema direto consiste na determinação do potencial elétrico $u$ no interior do domínio e da resposta $\overline{\mathbf{J}}$ no contorno, supondo-se conhecidas a distribuição de condutividade $\sigma$ e o estímulo $f$ aplicado neste contorno. Denotaremos o potencial $f$ aplicado por $u_{\text {aplic }}$. Com isso, pode-se resolver as equações (3-9), (3-10) e (3-11), gerando como resposta os valores dos fluxos de corrente $\overline{\mathbf{J}}$ medidos no contorno, que denotaremos por $\mathbf{J}_{\text {med }}$. Matematicamente, a resolução do problema direto pode ser vista como uma transformação dada através do seguinte operador

$$
\mathfrak{T}_{u_{\text {aplic }}}(\sigma)=\mathbf{J}_{\text {med }}
$$

que representa o processo de transformar as informações dadas por $u_{\text {aplic }}$ e $\sigma$ na resposta dada por $\mathbf{J}_{\text {med }}$.

Por sua vez, no problema da tomografia de impedância elétrica, a distribuição de condutividade $\sigma$ no interior do domínio $\Omega$ não é conhecida. As únicas informações que podem ser acessadas são os dados de estímulo-resposta 
no contorno do domínio. Então, devemos tratar o problema como um problema inverso que consiste na inversão do operador dado pela equação (3-12), ou seja, dados os valores do potencial $u_{\text {aplic }}$ aplicado e do fluxo de corrente $\mathbf{J}_{\text {med }}$ medido, determina-se a distribuição de condutividade $\sigma$ no interior do domínio:

$$
\mathfrak{T}_{u_{\text {aplic }}}^{-1}\left(\mathbf{J}_{\text {med }}\right)=\sigma
$$

Em resumo, o problema direto corresponde à resolução da equação (3-9) considerando-se conhecidas a distribuição de condutividade $\sigma$ e uma condição de contorno (equação (3-10)). Já o problema inverso consiste em resolver a equação (3-9) considerando-se conhecidas as duas condições de contorno (equações (3-10) e (3-11)), porém desconhecida a distribuição de condutividade $\sigma$ no interior do domínio.

Neste momento, podemos considerar termos em mãos o início de uma capa primitiva. Se quisermos esconder um objeto condutor dentro de $\Omega$, nós simplesmente escavamos um buraco não condutor para algum raio $\rho>0 \mathrm{em}$ $\Omega$ e colocamos o objeto dentro. O objeto é, assim, eletricamente isolado do exterior e não pode ser visto com a imagem de impedância. Infelizmente, o próprio buraco pode ser visto, por isso, um observador saberá que algo está sendo escondido, mesmo que ele não possa dizer o que é. No entanto, essa ideia é o início de uma camuflagem viável, mas primeiro precisamos analisar mais cuidadosamente a equação de Laplace em um anel $\Omega \backslash B_{\rho}(0)$.

\section{7}

\section{Solução para equação de Laplace no anel}

Vamos supor $D=B_{\rho}(0)$, semelhante ao painel central na figura 3.4, com $\rho<1$. Nosso objetivo é determinar $\rho$ usando imageamento por impedância. Uma maneira fácil de fazer isso é resolvendo a equação de Laplace com condições de fronteira (3-5) - (3-8) de forma explícita para ver que o valor de $\rho$ é, de fato, codificado nos dados de Neumann $\gamma \nabla u \cdot n$ em $\partial \Omega$. A solução para equação de Laplace, pode ser obtida com uma separação de variáveis em coordenadas polares que desenvolveremos abaixo.

No que segue, suponhamos $\gamma=1$, embora seja apenas por conveniência.

$\mathrm{O}$ domínio $\Omega \backslash D$ é um anel, por isso é conveniente escrever a equação de Laplace (3-4) em coordenadas polares

$$
\frac{\partial^{2} u}{\partial r^{2}}+\frac{1}{r} \frac{\partial u}{\partial r}+\frac{1}{r^{2}} \frac{\partial^{2} u}{\partial \theta^{2}}=0
$$

onde $u=u(r, \theta)$ é o potencial em $\Omega \backslash D$. Usando (3-14) é simples verificar que as soluções $1, \ln (r), r^{|k|} e^{\imath k \theta}$ e $r^{-|k|} e^{\imath k \theta}$, para $k \in \mathbb{Z}$, são harmônicas para 
$r>0$ e, consequentemente, sobre o anel $\Omega \backslash D$. Construiremos, assim, a solução relevante $u(r, \theta)$ com o argumento de superposição destas funções,

$$
u(r, \theta)=c_{0}+d_{0} \ln (r)+\sum_{k \in \mathbb{Z} \backslash\{0\}}\left(c_{k} r^{|k|}+d_{k} r^{-|k|}\right) e^{\imath k \theta}
$$

escolhendo corretamente os $c_{k}$ e $d_{k}$.

A condição de fronteira de Dirichlet $u=f$ em $\partial \Omega$ significa $u(1, \theta)=f(\theta)$, que é,

$$
c_{0}+\sum_{k \in \mathbb{Z} \backslash\{0\}}\left(c_{k}+d_{k}\right) e^{\imath k \theta}=f(\theta) \quad \text { para } \theta \in[0,2 \pi) .
$$

Isto parece a série de Fourier dos dados de Dirichlet $f$. Podemos assumir, então, que $f$ é bem comportada, isto é, contínua e diferenciável por partes, de modo que sua série de Fourier converge pontualmente para $f$. Neste sentido, podemos expandir $f$ em uma série de Fourier como

$$
f(\theta)=\sum_{k \in \mathbb{Z}} f_{k} e^{\imath k \theta} \text { onde } f_{k}=\frac{1}{2 \pi} \int_{0}^{2 \pi} f(\theta) e^{-\imath k \theta}
$$

Ao combinar os $f_{k}$ com os termos correspondentes à esquerda em (3-16), concluímos

$$
c_{0}=f_{0} \text { e } c_{k}+d_{k}=f_{k} \text { para } k \in \mathbb{Z} \backslash\{0\} .
$$

Para completar o cálculo, usamos as condições de fronteira de Neumann (3-8), que assume a forma $\frac{\partial u}{\partial r}=0$ em $\partial D$ (utilizando o fato de que o campo de vetores $n$ em $\partial D$ aponta radialmente em direção à origem, então $\frac{\partial}{\partial n}=\frac{-\partial}{\partial r}$ ao longo desta fronteira interna. Formalmente, tomando a derivada termo a termo de (3-15) em relação à $r$ e avaliando em $r=\rho$ temos

$$
\frac{d_{0}}{\rho}+\sum_{k \in \mathbb{Z} \backslash\{0\}}|k|\left(c_{k} \rho^{|k|-1}-d_{k} \rho^{-|k|-1}\right) e^{\imath k \theta}=0 \text { para } \theta \in[0,2 \pi) .
$$

A equação (3-18) pode ser interpretada como a série de Fourier da função identicamente nula, cujos coeficientes de Fourier são iguais a zero, por isso, podemos concluir que

$$
d_{0}=0 \text { e }|k|\left(c_{k} \rho^{|k|-1}-d_{k} \rho^{-|k|-1}\right)=0 \text { para } k \in \mathbb{Z} \backslash\{0\} .
$$

Resolvendo (3-17) e (3-19) para $c_{k}$ e $d_{k}$, e substituindo em (3-15) para se obter a solução para a equação de Laplace no anel que satisfaça as condições de 
fronteira $(3-5)$ e (3-8):

$$
u(r, \theta)=\sum_{k \in \mathbb{Z}}\left(\frac{f_{k}}{1+\rho^{2|k|}} r^{|k|} e^{\imath k \theta}+\frac{\rho^{2|k|} f_{k}}{1+\rho^{2|k|}} r^{-|k|} e^{\imath k \theta}\right)
$$

para todo $r \in[\rho, 1]$ e $\theta \in[0,2 \pi) ; u$ é indefinida dentro de $D$. A partir de (3-20), podemos facilmente calcular os dados de Neumann em $\partial \Omega$, observando que $\frac{\partial}{\partial n}=\frac{\partial}{\partial r}$ nesta fronteira externa, onde $r=1$ :

$$
\frac{\partial u}{\partial n}(1, \theta)=\sum_{k \in \mathbb{Z}} \frac{|k|\left(1-\rho^{2|k|}\right)}{1+\rho^{2|k|}} f_{k} e^{\imath k \theta}
$$

Podemos calcular a solução para a equação de Laplace no disco aberto (sem o "vácuo" D) usando o mesmo procedimento, mas omitindo os termos $\ln (r) \mathrm{e}$ $r^{-|k|} e^{\imath k \theta}$ em (3-15). Como se poderia esperar, a solução acaba sendo exatamente o que se obtém a partir de (3-20) com $\rho=0$. A mesma observação vale para os dados de Neumann em (3-21). Deve-se ressaltar que é preciso assumir que $f$ é suave o suficiente para que a série de Fourier (3-21) convirja de forma significativa, por exemplo, pontualmente para alguma função contínua.

Nesse contexto, vejamos no exemplo a seguir como o raio $\rho$ do buraco pode ser codificado através da informação obtida com os dados de Neumman.

Exemplo 3.7.1 Podemos determinar o potencial $u(r, \theta)$ no anel $\rho \leq r \leq 1$ que satisfaz $u(1, \theta)=\cos \theta$ e $\frac{\partial u}{\partial n}(\rho, \theta)=0$, e em seguida calcular $\frac{\bar{\partial} u}{\partial n}(1, \theta)$, vendo, assim, como o raio $\rho$ do buraco é codificado nesta informação.

Temos, a priori, $u(r, \theta)=\frac{\left(r^{2}+\rho^{2}\right)}{r\left(1+\rho^{2}\right)} \cos \theta$. Note que, $f_{-1}=\overline{f_{1}}=\frac{1}{2}$, pois $f=\cos \theta$ é real, $f_{0}=0$, e $u(1, \theta)=\cos \theta$. Além disso, $\frac{\partial u}{\partial n}(\rho, \theta)=\frac{\partial u}{\partial r}(\rho, \theta)=$ $\frac{\left(\rho^{2}-\rho^{2}\right)}{\rho^{2}\left(1+\rho^{2}\right)} \cos \theta=0, e \frac{\partial u}{\partial n}(1, \theta)=\frac{\left(1-\rho^{2}\right)}{1+\rho^{2}} \cos \theta$.

\section{8}

\section{Capa Inadequada}

Como mencionado acima, a maneira pela qual podemos tentar ocultar um objeto dentro de $\Omega$ é abrindo um espaço $D=B_{\rho}(0)$ para algum $0<\rho<1$ adequado, dispondo ali o objeto, isolando-o, assim, eletricamente de $\partial \Omega$. O observador não pode reunir qualquer informação sobre o objeto, uma vez que os dados de Neumann são dados por (3-21) e não dependem do que está dentro de $D$. Infelizmente, a expressão em (3-21) mostra que os dados de Neumann à direita são claramente dependentes de $\rho$. Se $\rho>0$, o observador provavelmente estará ciente de que algo suspeito está acontecendo. 
Para quantificar isso, denotaremos por $u_{0}$ a solução para a equação de Laplace em $\Omega$ com dados de Dirichlet $u_{0}=f$, quando nenhum vácuo $D$ está presente ( $\Omega$ está vazio). Seja $u$ a solução em $\Omega \backslash D$ com $D=B_{\rho}(0), u=f$ em $\partial \Omega$, e a condição de fronteira (3-8). Queremos calcular apenas quanto os dados de Neumann para $u$ e $u_{0}$ diferem em termos de $\rho$. A diferença nos dados de Neumann para $u$ e $u_{0}$ é, a partir de (3-21),

$$
\begin{aligned}
\frac{\partial u}{\partial n}(1, \theta)-\frac{\partial u_{0}}{\partial n}(1, \theta) & =\sum_{k \in \mathbb{Z}} \frac{|k|\left(1-\rho^{2|k|}\right)}{1+\rho^{2|k|}} f_{k} e^{\imath k \theta}-\sum_{k \in \mathbb{Z}}|k| f_{k} e^{\imath k \theta} \\
& =-\sum_{k \in \mathbb{Z}} \frac{2|k| \rho^{2|k|}}{1+\rho^{2|k|}} f_{k} e^{\imath k \theta}
\end{aligned}
$$

Uma maneira conveniente de medir o valor da diferença é considerar a norma de $L^{2}(\partial \Omega)$ :

$$
\left\|\frac{\partial u}{\partial n}(1, \theta)-\frac{\partial u_{0}}{\partial n}(1, \theta)\right\|_{L^{2}(\partial \Omega)}^{2}:=\int_{0}^{2 \pi}\left|\frac{\partial u}{\partial n}(1, \theta)-\frac{\partial u_{0}}{\partial n}(1, \theta)\right|^{2} d \theta
$$

$$
\int_{0}^{2 \pi}\left|\frac{\partial u}{\partial n}(1, \theta)-\frac{\partial u_{0}}{\partial n}(1, \theta)\right|^{2} d \theta=2 \pi \sum_{k \in \mathbb{Z}} \frac{4 k^{2} \rho^{4|k|}}{\left(1+\rho^{2|k|}\right)^{2}}\left|f_{k}\right|^{2}
$$

em que a última linha decorre de (3-22) e identidade de Parseval (ver apêndice). De $(3-23)$ e do fato que $\frac{\rho^{4|k|}}{\left(1+\rho^{2|k|}\right)^{2}}<\rho^{4}$ se $0<\rho<1$ e $|k| \geq 1$, vemos que

$$
\begin{aligned}
\left\|\frac{\partial u}{\partial n}(1, \theta)-\frac{\partial u_{0}}{\partial n}(1, \theta)\right\|_{L^{2}(\partial \Omega)}^{2} & =2 \pi \sum_{k \in \mathbb{Z}} \frac{4 k^{2} \rho^{4|k|}}{\left(1+\rho^{2|k|}\right)^{2}}\left|f_{k}\right|^{2} \\
& \leq 8 \pi \sum_{k \in \mathbb{Z}} k^{2} \rho^{4}\left|f_{k}\right|^{2}=4 \rho^{4}\left(2 \pi \sum_{k \in \mathbb{Z}} k^{2}\left|f_{k}\right|^{2}\right) \\
& =4 \rho^{4}\left\|\frac{\partial u_{0}}{\partial n}(1, \theta)\right\|_{L^{2}(\partial \Omega)}^{2}
\end{aligned}
$$

A última igualdade no lado direito acima segue por considerarmos $\rho=0$ em (3-21) e usando a identidade de Parseval. Tomando a raiz quadrada de cada expressão acima nos leva à:

$$
\left\|\frac{\partial u}{\partial n}(1, \theta)-\frac{\partial u_{0}}{\partial n}(1, \theta)\right\|_{L^{2}(\partial \Omega)} \leq 2 \rho^{2}\left\|\frac{\partial u_{0}}{\partial n}(1, \theta)\right\|_{L^{2}(\partial \Omega)}
$$

Em suma, se o orifício for pequeno, a diferença entre os dados de Neumann serão pequenos, proporcional a $\rho^{2}$ (isto é, à área do orifício). Se o observador mede os dados de Neumann com precisão finita, podemos esconder o objeto, fazendo $\rho$ tão pequeno que perturba os dados de Neumann a um nível abaixo 
do limite de precisão - mas somente se o objeto se encaixar! Se o observador fizer as medições dos dados de Neumann em precisão alta o suficiente, então (3-24) ditará um valor para $\rho$ muito pequeno para esconder nosso objeto, e, assim, essa abordagem não funcionará.

Com essas ideias, vejamos através do exemplo abaixo quanto os dados de Neumann para $u$ e $u_{0}$ diferem em termos de $\rho$. Consideremos para tanto $u$ satisfazendo as condições como no exemplo 3.7.1.

Exemplo 3.8.1 Podemos calcular $\left\|\frac{\partial u}{\partial n}(1, \theta)-\frac{\partial u_{0}}{\partial n}(1, \theta)\right\|_{L^{2}(\partial \Omega)}$ sendo o dado de Dirichlet $f(\theta)=\cos \theta$.

Inicialmente, observemos que $u_{0}(r, \theta)=r \cos \theta, u(r, \theta)=\frac{\left(r^{2}-\rho^{2}\right)}{r\left(1+\rho^{2}\right)} \cos \theta$, e, assim, $\frac{\partial u_{0}}{\partial n}(1, \theta)=\cos \theta, \frac{\partial u}{\partial n}(1, \theta)=\frac{\left(1-\rho^{2}\right)}{\left(1+\rho^{2}\right)} \cos \theta$. Logo,

$$
\begin{aligned}
\left\|\frac{\left(1-\rho^{2}\right)}{1+\rho^{2}} \cos \theta-\cos \theta\right\|_{L_{(\partial \Omega)}^{2}}^{2} & =\int_{0}^{2 \pi}\left|\frac{\left(1-\rho^{2}\right)}{1+\rho^{2}} \cos \theta-\cos \theta\right|^{2} d \theta \\
& =\int_{0}^{2 \pi}\left(\frac{-2 \rho^{2}}{1+\rho^{2}} \cos \theta\right)^{2} d \theta \\
& =\frac{4 \rho^{4}}{\left(1+\rho^{2}\right)^{2}} \int_{0}^{2 \pi} \cos ^{2} \theta d \theta \\
& =\frac{4 \rho^{4}}{\left(1+\rho^{2}\right)^{2}} \int_{0}^{2 \pi} \frac{(1+\cos 2 \theta)}{2} d \theta=\frac{4 \rho^{4}}{\left(1+\rho^{2}\right)^{2}} \pi
\end{aligned}
$$

Daí, $\left\|\frac{\partial u}{\partial n}(1, \theta)-\frac{\partial u_{0}}{\partial n}(1, \theta)\right\|_{L^{2}(\partial \Omega)}=\left\|\frac{\left(1-\rho^{2}\right)}{1+\rho^{2}} \cos \theta-\cos \theta\right\|_{L_{(\partial \Omega)}^{2}}=\frac{2 \rho^{2}}{1+\rho^{2}} \sqrt{\pi}$.

Pode-se ainda pensar em uma generalização para o exemplo 3.7 .1 com o seguinte:

Exemplo 3.8.2 Se o coeficiente de Fourier $f_{1}$ é não nulo, então podemos, em princípio, determinar $\rho$, a partir da fronteira dada, avaliando a integral

$$
I=\int_{0}^{2 \pi} \frac{\partial u}{\partial n}(1, \theta) e^{-\imath \theta} d \theta
$$

e então resolvendo $\frac{\left(1-\rho^{2}\right)}{\left(1+\rho^{2}\right)}=\frac{I}{2 \pi f_{1}}$ para $\rho$ (note que $f_{1}$ está bem definida a partir dos dados de Dirichlet).

Com efeito, usando (3-21) e o argumento de ortogonalidade das funções $e^{\imath k \theta}$ em $[0,2 \pi]$, isto é,

$$
\int_{0}^{2 \pi} e^{\imath n \theta} e^{\imath m \theta}=\left\{\begin{array}{lll}
2 \pi, & \text { se } & n=-m \\
0, & \text { se } & n \neq-m
\end{array}\right.
$$

temos 


$$
\int_{0}^{2 \pi} e^{\imath k \theta} e^{-\imath \theta}=\left\{\begin{array}{lll}
2 \pi, & \text { se } & k=1 \\
0, & \text { se } & k \neq 1
\end{array}\right.
$$

e, logo obtemos

$$
\begin{aligned}
I & =\int_{0}^{2 \pi}\left(\frac{\partial u}{\partial n}(1, \theta) e^{-\imath \theta} d \theta\right. \\
& =\int_{0}^{2 \pi}\left(\sum_{k \in \mathbb{Z}} \frac{|k|\left(1-\rho^{2|k|}\right)}{1+\rho^{2|k|}} f_{k} e^{\imath k \theta}\right) e^{-\imath \theta} d \theta \\
& =\sum_{k \in \mathbb{Z}} \frac{|k|\left(1-\rho^{2|k|}\right)}{1+\rho^{2|k|}} f_{k} \int_{0}^{2 \pi} e^{\imath k \theta} e^{-\imath \theta} d \theta=\frac{\left(1-\rho^{2}\right)}{1+\rho^{2}} f_{1} 2 \pi
\end{aligned}
$$

Portanto, como $f_{1}$ é não nulo, (3-25) resulta em $\frac{\left(1-\rho^{2}\right)}{1+\rho^{2}}=\frac{I}{2 \pi f_{1}}$

Neste contexto, quanto os dados de Neumann diferem em termos de $\rho$ ? O seguinte exemplo nos mostrará que, de fato, os dados de Neumann para $u$ e $u_{0}$ devem diferir em pelo menos uma quantidade proporcional a $\rho^{2}$.

Exemplo 3.8.3 Se o coeficiente de Fourier $f_{1}$ é não nulo (e note que $f_{-1}=\overline{f_{1}}$ se $f$ é real), então

$$
\left\|\frac{\partial u}{\partial n}(1, \theta)-\frac{\partial u_{0}}{\partial n}(1, \theta)\right\|_{L^{2}(\partial \Omega)} \geq \frac{4 \sqrt{\pi} \rho 2\left|f_{1}\right|}{1+\rho^{2}} \geq 2 \sqrt{\pi}\left|f_{1}\right| \rho^{2}
$$

$\operatorname{para} \rho \leq 1$

Consideremos que $f$ é real e, portanto, $f_{-1}=\overline{f_{1}}$. Logo, $\left|f_{-1}\right|=\left|f_{1}\right|$.

Temos, assim, que

$$
\begin{aligned}
\left\|\frac{\partial u}{\partial n}(1, \theta)-\frac{\partial u_{0}}{\partial n}(1, \theta)\right\|_{L^{2}(\partial \Omega)}^{2} & =2 \pi \sum_{k \in \mathbb{Z}} \frac{4 k^{2} \rho^{4|k|}}{\left(1+\rho^{2|k|}\right)^{2}}\left|f_{k}\right|^{2} \\
& \geq 2 \pi \frac{4 \rho^{4}\left|f_{-1}\right|^{2}}{\left(1+\rho^{2}\right)^{2}}+2 \pi \frac{4 \rho^{4}\left|f_{1}\right|^{2}}{\left(1+\rho^{2}\right)^{2}}=\frac{16 \pi\left|f_{1}\right|^{2} \rho^{4}}{\left(1+\rho^{2}\right)^{2}}
\end{aligned}
$$

logo, extraindo a raiz quadrada em ambos os membros da desigualdade acima, chegamos a $\left\|\frac{\partial u}{\partial n}(1, \theta)-\frac{\partial u_{0}}{\partial n}(1, \theta)\right\|_{L^{2}(\partial \Omega)} \geq \frac{4 \sqrt{\pi}\left|f_{1}\right| \rho^{2}}{\left(1+\rho^{2}\right)} \geq 2 \sqrt{\pi}\left|f_{1}\right| \rho^{2}$, para $\rho \leq 1$. 


\section{Construção da Camuflagem}

O que precisamos é uma maneira de inserir um grande buraco em $\Omega$ enquanto fazemos com que pareça um buraco muito pequeno para um observador externo, ou simplesmente, nenhum! Mostraremos como fazer isso no caso $D=B_{\frac{1}{2}}(0)$, embora funcione para um orifício de qualquer raio menor que 1. A chave é cercar o buraco $D$ com um anel de material que tenha uma condutividade anisotrópica adequada. As propriedades desejadas desta condutividade anisotrópica podem ser deduzidas a partir de um simples argumento de mudança de variáveis.

4.1

\section{Mudança de Variáveis}

Usaremos $\Omega_{\rho}$ para denotar o anel aberto $\Omega \backslash \bar{B}_{\rho}(0)$ (a linha superior indica o fecho da bola). Escolha $\rho \in\left(0, \frac{1}{2}\right)$ e seja $u$ uma solução duas vezes continuamente diferenciável para a equação de Laplace em $\Omega_{\rho}$, com dados de Dirichlet $f$ em $\partial \Omega$ e condição de fronteira de isolamento (3-8). Seja $\phi$ uma função invertível de $\bar{\Omega}_{\rho}$ em $\bar{\Omega}_{\frac{1}{2}}$, suponha que $\phi$ e $\phi^{-1}$ sendo duas vezes continuamente diferenciáveis. Denotaremos por $x=\left(x_{1}, x_{2}\right)$ as coordenadas retangulares em $\Omega_{\rho}$ e $y=\left(y_{1}, y_{2}\right)$ as coordenadas retangulares em $\Omega_{\frac{1}{2}}$, com $y=\phi(x)$. Assuma que $\phi$ leva a fronteira interna $\|x\|=\rho$ de $\Omega_{\rho}$ na fronteira interna $\|y\|=\frac{1}{2}$ de $\Omega_{\frac{1}{2}}$, $\phi$ também leva $\|x\|=1$ em $\|y\|=1$, e que a derivada de $\phi$

$$
\mathbf{D} \phi(x)=\left(\begin{array}{ll}
\frac{\partial y_{1}}{\partial x_{1}} & \frac{\partial y_{1}}{\partial x_{2}} \\
\frac{\partial y_{2}}{\partial x_{1}} & \frac{\partial y_{2}}{\partial x_{1}}
\end{array}\right)
$$

é não singular em $\bar{\Omega}_{\rho}$.

Defina uma função $v$ em $\Omega_{\frac{1}{2}}$ por $v(y)=u\left(\phi^{-1}(y)\right)$, ou equivalentemente, $v(\phi(x))=u(x)$. Ou seja, $v$ é simplesmente a função "pushed forward" de $\Omega_{\rho}$ para o domínio $\Omega_{\frac{1}{2}}$ pela função $\phi$.

Como $\Delta u=0$ em $\Omega_{\rho}, v$ satisfaz uma certa equação diferencial em $\Omega_{\frac{1}{2}}$ o foco do seguinte lema. 
Lema 4.1.1 Com base nos pressupostos acima, a função v(y) satisfaz a equação diferencial parcial:

$$
\nabla \cdot(\sigma(y) \nabla v)=0
$$

em $\Omega_{\frac{1}{2}}$, onde $\sigma(y)$ denota a matriz $2 \times 2$

$$
\sigma(y)=\frac{D \phi(x)(D \phi(x))^{T}}{|\operatorname{det}(D \phi(x))|}
$$

avaliado em $x=\phi^{-1}(y)$.

Prova: Certamente a prova deste lema pode ser feita aplicando-se o Laplaciano em $x$ para os dois lados da equação $u(x)=v(\phi(x))$ e usando a regra da cadeia, porém, torna-se trabalhoso e confuso. A maneira mais elegante é obter a prova através do teorema da divergência. Inicialmente, a regra da cadeia aplicada a $u(x)=v(\phi(x))$ produz

$$
\begin{aligned}
\frac{\partial u}{\partial x_{1}}(x) & =\frac{\partial y_{1}}{\partial x_{1}} \frac{\partial v}{\partial y_{1}}(\phi(x))+\frac{\partial y_{2}}{\partial x_{1}} \frac{\partial v}{\partial y_{2}}(\phi(x)), \\
\frac{\partial u}{\partial x_{2}}(x) & =\frac{\partial y_{1}}{\partial x_{2}} \frac{\partial v}{\partial y_{1}}(\phi(x))+\frac{\partial y_{2}}{\partial x_{2}} \frac{\partial v}{\partial y_{2}}(\phi(x)) .
\end{aligned}
$$

Essas equações podem ser escritas de forma mais compacta como $\nabla_{x} u(x)=$ $(D \phi(x))^{T} \nabla_{y} v(\phi(x))$, onde $D \phi$ é como acima definido, $\nabla_{x}$ refere-se ao gradiente em $\left(x_{1}, x_{2}\right)$ e $\nabla_{y}$ refere-se ao gradiente em $\left(y_{1}, y_{2}\right)$.

Seja $w(x)$ uma função continuamente diferenciável definida em $\bar{\Omega}_{\rho}$ com $w=0$ em $\partial \Omega_{\rho}$, e defina $\widetilde{w}$ em $\Omega_{\frac{1}{2}}$ através de $\widetilde{w}(y)=w\left(\phi^{-1}(y)\right)($ ou $w(x)=\widetilde{w}(\phi(x))$. Assim, vale que $\nabla_{x} w(x)=(D \phi(x))^{T} \nabla_{y} \widetilde{w}(\phi(x))$. Como $\Delta_{x} u=0$ em $\Omega_{\rho}\left(\Delta_{x}\right.$ é o laplaciano nas coordenadas $\left.x\right)$ temos:

$$
\int_{\Omega_{\rho}} w(x) \Delta_{x} u(x) d x=0
$$

Note que $w \Delta_{x} u=\nabla_{x} \cdot\left(w \nabla_{x} u\right)-\nabla_{x} w \nabla_{x} u$. Logo, substituindo em (4-3) e aplicando o teorema da divergência para o primeiro termo, obtemos:

$$
\int_{\partial \Omega_{\rho}} w \nabla_{x} u \cdot n d s-\int_{\Omega_{\rho}} \nabla_{x} w \cdot \nabla_{x} u d x=0 .
$$

Como $w \equiv 0$ em $\partial \Omega_{\rho}$, a primeira integral acima é igual a zero, então:

$$
\int_{\Omega_{\rho}}\left(\nabla_{x} w\right)^{T} \nabla_{x} u d x=0
$$


já que $\nabla_{x} w \cdot \nabla_{x} u=\left(\nabla_{x} w\right)^{T} \nabla_{x} u$. Logo, como $\nabla_{x} u(x)=(D \phi(x))^{T} \nabla_{y} v(\phi(x))$ e $\nabla_{x} w(x)=(D \phi(x))^{T} \nabla_{y} \widetilde{w}(\phi(x))$, podemos escrever a última equação como:

$$
\int_{\Omega_{\rho}} \nabla_{y} \widetilde{w}(\phi(x))^{T}(D \phi(x))(D \phi(x))^{T} \nabla_{y} v(\phi(x)) d x=0 .
$$

Agora, fazendo uma mudança de variáveis para o sistema de coordenadas $y$, $\operatorname{com} \phi(x)=y$ e $d x=\frac{d y}{|\operatorname{det}(D \phi)|}$, encontramos:

$$
\int_{\Omega_{\frac{1}{2}}}\left(\nabla_{y} \widetilde{w}(y)\right)^{T}\left(\sigma(y) \nabla_{y} v(y)\right) d y=0
$$

com $\sigma(y)$ definida como no enucniado do lema. Além disso, temos que:

$$
\begin{aligned}
\left(\nabla_{y} \widetilde{w}(y)\right)^{T}\left(\sigma(y) \nabla_{y} v(y)\right) & = \\
=\nabla_{y} \widetilde{w}(y) \cdot\left(\sigma(y) \nabla_{y} v(y)\right) & = \\
=\left(\nabla_{y} \widetilde{w}(y)\right)\left(\sigma(y) \nabla_{y} v(y)\right)+\widetilde{w}(y) \nabla_{y} \cdot\left(\sigma(y) \nabla_{y} v(y)\right) & = \\
=\nabla_{y} \cdot\left(\widetilde{w}(y) \sigma(y) \nabla_{y} v(y)\right)-\widetilde{w}(y) \nabla_{y} \cdot\left(\sigma(y) \nabla_{y} v(y)\right) . &
\end{aligned}
$$

Substituindo (4-5) no integrando em (4-4), encontramos

$$
\int_{\Omega_{\frac{1}{2}}} \nabla_{y} \cdot\left(\widetilde{w}(y) \sigma(y) \nabla_{y} v(y)\right) d y-\int_{\Omega_{\frac{1}{2}}} \widetilde{w}(y) \nabla_{y} \cdot\left(\sigma(y) \nabla_{y} v(y)\right) d y=0 .
$$

Logo, pelo teorema da divergência, para a primeira integral do lado esquerdo acima, e pelo fato de que $\widetilde{w} \equiv 0$ em $\partial \Omega_{\frac{1}{2}}$, segue que esta integral é igual a zero, e, assim, obtemos

$$
\int_{\Omega_{\frac{1}{2}}} \widetilde{w}(y) \nabla_{y} \cdot\left(\sigma(y) \nabla_{y} v(y)\right) d y=0
$$

A função $\widetilde{w}(y)$ é arbitrária (já que dado qualquer $\widetilde{w}$ poderíamos ter escolhido $w(x)=\widetilde{w}\left(\phi^{-1}(x)\right)$ tomado sobre $\left.\Omega_{\rho}\right)$, então (4-6) vale para qualquer $\widetilde{w}$ continuamente diferenciável. Afirmamos que isto força $\nabla_{y} \cdot\left(\sigma(y) \nabla_{y} v(y)\right)$ a ser identicamente nula em $\Omega_{\frac{1}{2}}$.

Com efeito, seja $h(y)=\nabla_{y} \cdot\left(\sigma(y) \nabla_{y} v(y)\right)$. Note que, a partir dos pressupostos em $\phi$ e $u$, a função $h$ é contínua em $\Omega_{\frac{1}{2}}$. Suponhamos que, em contradição com a afirmação de que $h$ é identicamente nula em $\Omega_{\frac{1}{2}}, h\left(y_{0}\right)>0$ para algum ponto $y_{0}$. Como $h$ é contínua, temos que $h(y)>0$ qualquer que seja $y \in B_{\delta}\left(y_{0}\right) \subset \Omega_{\frac{1}{2}}$ para algum $\delta>0$. Podemos escolher uma função $\widetilde{w}(y) \geq 0$ que é positiva em $B_{\delta}\left(y_{0}\right)$ e $\widetilde{w}(y) \equiv 0$ fora de $B_{\delta}\left(y_{0}\right)$. Como resultado, o produto 
$\widetilde{w}(y) h(y) \geq 0$ em $\Omega_{\frac{1}{2}}$ e $\widetilde{w}(y) h(y)$ é não identicamente nula. Mas, então, a integral em (4-6) não pode ser igual a zero, uma contradição. Conclui-se que $h(y)=\nabla_{y} \cdot\left(\sigma(y) \nabla_{y} v(y)\right)=0$ em $\Omega_{\frac{1}{2}}$, e isto prova o lema.

A comparação de (4-1) a (3-7) mostra que $v$ pode ser considerado como o potencial elétrico dentro de $\Omega_{\frac{1}{2}}$ correspondente a condutividade anisotrópica $\sigma$. É esta observação que nos permitirá projetar uma condutividade anisotrópica para encobrir a bola $B_{\frac{1}{2}}(0)$. A proposição a seguir nos fornecerá algumas informações relevantes sobre a matriz $\sigma$.

Proposição 4.1.2 A matriz $\sigma(y)$ definida por (4-2) é simétrica e positiva definida para cada $y$, isto é, satisfaz $w^{T} \sigma(y) w>0$ para cada vetor $w \in \mathbb{R}^{2}$ não nulo.

Prova: Com efeito, fixemos $y=\phi(x)$ para algum $x \in \bar{\Omega}_{\rho}$, e denotemos por $D \phi(x):=D$ e $\sigma(y):=\sigma=\frac{D D^{T}}{\operatorname{det}(D)}$. Logo, $\sigma^{T}=\frac{1}{\operatorname{det}(D)}\left(D D^{T}\right)^{T}=$ $\frac{1}{\operatorname{det}(D)}\left(D^{T}\right)^{T}(D)^{T}=\frac{1}{\operatorname{det}(D)} D D^{T}=\sigma$. Daí, $\sigma^{T}=\sigma$ mostrando que $\sigma$ é simétrica. Além disso, podemos mostrar que se $w \neq \overrightarrow{0}$ então $w^{T} \sigma w>0$. De fato, seja $v=D^{T} w$, para $w$ não nulo. Então, $w^{T} \sigma w=w^{T} \frac{\left(D D^{T}\right)}{|\operatorname{det}(D)|} w=$ $\frac{1}{|\operatorname{det}(D)|} v^{T} v$. Como $w \neq \overrightarrow{0}, D$ é não singular e $v^{T} v>0$ segue que $w^{T} \sigma w=$ $\frac{1}{|\operatorname{det}(D)|} v^{T} v>0$, concluindo que $\sigma$ é positiva definida. Portanto, como $y \in \bar{\Omega}_{\frac{1}{2}}$ é arbitrário, segue que $\sigma(y)$ é simétrica e positiva definida para cada $y$.

\section{2}

\section{Criando a capa}

As propriedades necessárias a partir da camada do material anisotrópico em volta de $D=B_{\frac{1}{2}}(0)$ podem ser deduzidas ao considerarmos funções $\phi: \Omega_{\rho} \rightarrow \Omega_{\frac{1}{2}}$ com a seguinte fórmula:

$$
\phi(x)=\frac{\psi(\|x\|)}{\|x\|} x
$$

tal que $y=\phi(x)$ signifique $y_{1}=\frac{\psi(\|x\|)}{\|x\|} x_{1}$ e $y_{2}=\frac{\psi(\|x\|)}{\|x\|} x_{2}$, onde $\psi$ é uma função escolhida de maneira que:

1. $\psi(\rho)=\frac{1}{2}\left(\phi\right.$ leva a fronteira interna de $\Omega_{\rho}$ na de $\left.\Omega_{\frac{1}{2}}\right)$;

2. para um $\delta \in\left(0, \frac{1}{2}\right)$ temos $\psi(r)=r$ para $\frac{1}{2}+\delta<r<1$ (então $\phi$ fixa uma vizinhança $\frac{1}{2}+\delta<\|x\| \leq 1$ de outra fronteira em $\|x\|=1$ ); 
3. A função $\psi$ é duas vezes continuamente diferenciável, com $\psi^{\prime}(r) \geq d_{0}>0$ para algum $d_{0}$, então $\psi$ será estritamente crescente e invertível.

O mapeamento $\phi$ simplesmente "empurra" pontos de $\Omega_{\rho}$ radialmente para fora da origem, pelo menos para $\rho \leq\|x\|<\frac{1}{2}+\delta$. Há muitas maneiras de fraudar essa $\psi$, por exemplo,

$$
\psi(r)= \begin{cases}\frac{1}{2}+\frac{\delta}{1-2 \rho}(r-\rho), & \rho \leq r \leq \frac{1}{2} \\ g(r), & \frac{1}{2}<r<\frac{1}{2}+\delta \\ r, & \frac{1}{2}+\delta \leq r \leq 1\end{cases}
$$

onde $g(r)$ é uma função escolhida adequadamente para interpolar suavemente entre as duas regiões em que $\psi$ é uma reta. A fórmula precisa para $g$ não é importante no momento. Um típico $\psi$ e o mapeamento resultante de $\Omega_{\rho}$ para $\Omega_{\frac{1}{2}}$ é mostrado na figura 4.1 .

Sob tal mapeamento $\phi$ temos $y=x$ em uma vizinhança $\frac{1}{2}+\delta \leq r \leq 1$ da fronteira exterior, e então $u \equiv v$ nesta região. A função $v=u \circ \phi^{-1}$ também tem dados de Neumann zero na fronteira interior $\|y\|=\frac{1}{2}$. Especificamente, temos:

$$
\begin{aligned}
\left.\frac{\partial v}{\partial n}\right|_{\|y\|=\frac{1}{2}} & \left.=-\left.\frac{\partial v}{\partial\|y\|}\right|_{\|y\|=\frac{1}{2}} \text { (lembre que } \frac{\partial}{\partial n}=-\frac{\partial}{\partial\|y\|} \text { em }\|y\|=\frac{1}{2}\right) \\
& =-\left.\frac{\partial\|x\|}{\partial\|y\|} \frac{\partial u}{\partial\|x\|}\right|_{\|x\|=\rho} \\
& =-\left.\frac{(1-2 \rho)}{\delta} \frac{\partial u}{\partial\|x\|}\right|_{\|x\|=\rho} \\
& =0
\end{aligned}
$$

onde utilizamos $\|y\|=\psi(\|x\|)$ e o primeiro caso em (4-8), que produz $\frac{\partial\|y\|}{\partial\|x\|}=\frac{\delta}{(1-2 \rho)}$ em $\|x\|=\rho$, por isso $\frac{\partial\|x\|}{\partial\|y\|}=\frac{(1-2 \rho)}{\delta}$.

O leitor interessado encontrará no apêndice um exemplo de como se obter uma típica função $\psi$ construindo uma interpolação através da fórmula de Taylor. 

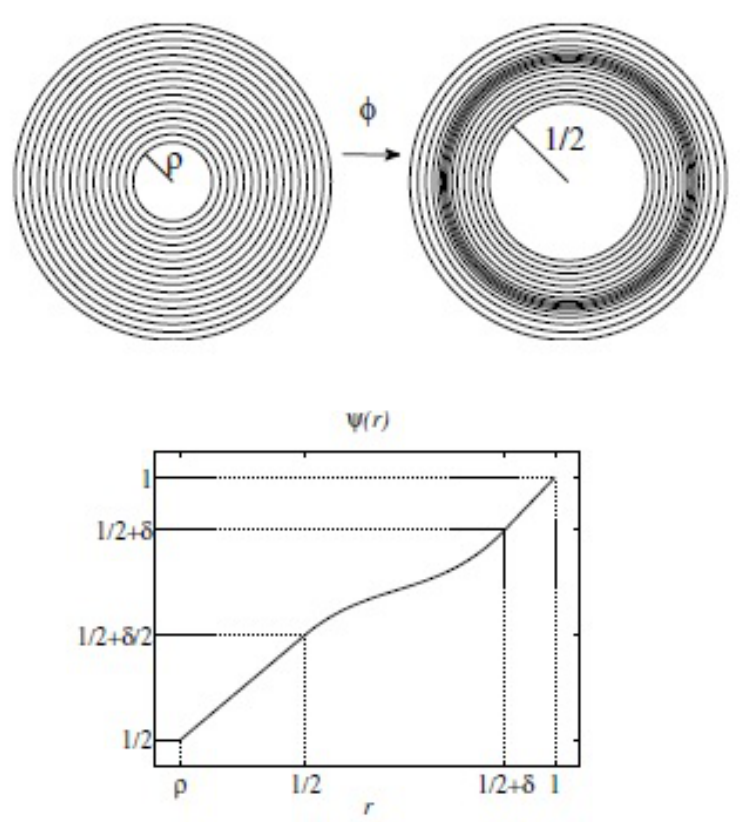

Figura 4.1: Um exemplo de uma função $\phi(\mathbf{x})=\frac{\psi(\|\mathbf{x}\|)}{\|\mathbf{x}\|} \mathbf{x}$, onde $\psi(r)$ é definida via (4-8). Note que $\phi$ leva o círculo de raio $r$ no círculo de raio $\psi(r)$.

\section{3}

\section{A condutividade $\sigma$ é uma camuflagem aproximada}

Afirmamos que a condutividade $\sigma$ definida por (4-2) pode ser usada para encobrir o vácuo $D$ em qualquer grau desejado, com $\rho$ sendo um parâmetro que controla a qualidade da camuflagem. Para isso, note que a matriz $\sigma$ corresponde a escala de condutividade 1 em $\Omega_{\frac{1}{2}}$ quando $\|y\|>\frac{1}{2}+\delta$, isto é, numa vizinhança da fronteira exterior e, como observado acima, $v$ e $u$ são iguais nesta região. Isso significa que $u$ e $v$ têm precisamente os mesmos dados de Dirichlet e Neumann em $\partial \Omega$. Na "região de camuflagem" $\frac{1}{2}<\|y\|<\frac{1}{2}+\delta$, a quantidade $\sigma(y)$ corresponde a uma condutividade anisotrópica. À luz da estimativa (3-24) e $\frac{\partial v}{\partial n}=\frac{\partial u}{\partial n}$ em $\partial \Omega$ vemos que

$$
\left\|\frac{\partial v}{\partial n}(1, \theta)-\frac{\partial u_{0}}{\partial n}(1, \theta)\right\|_{L^{2}(\partial \Omega)}=\rho^{2}\left\|\frac{\partial u_{0}}{\partial n}(1, \theta)\right\|_{L^{2}(\partial \Omega)}
$$

mesmo que $v$ seja o potencial de uma região $\Omega_{\frac{1}{2}}$ com um orifício central com um raio $\frac{1}{2}$. Fazendo $\rho$ tender a zero, podemos tornar os dados de Neumann para $v$ tão próximos dos dados de Neumann para $u_{0}$ quanto queiramos - é possível tornarmos a região com buraco de tamanho $\frac{1}{2}$ parecer tão vazia como gostaríamos! Veja a figura 4.2 como exemplo. 

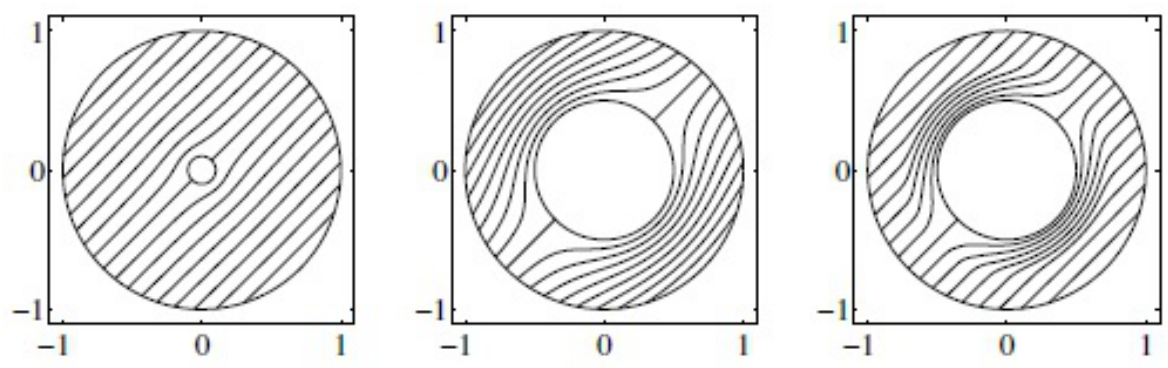

Figura 4.2: Comparação de soluções descamufladas e camufladas nos anéis. Os gráficos da esquerda e do meio mostram o fluxo das linhas de corrente $\mathbf{J}=-\gamma \nabla u$, onde $u$ é uma solução da equação de Laplace com condições de Dirichlet $f(\theta)=\cos (\theta)+\sin (\theta)$ (o potencial aplicado pelo observador) na fronteira exterior dos anéis com condutividade constante. $\mathrm{O}$ gráfico à direita mostra fluxos de linha da corrente $\mathbf{J}=-\gamma \nabla v$ para o círculo aproximadamente camuflado com condutividade anisotrópica $\sigma$ correspondente a $\rho=\frac{1}{10}$.

\section{4}

\section{Comportamento na região de camuflagem}

É extremamente interessante examinar o comportamento de $\sigma$ no interior da região de camuflagem $\frac{1}{2} \leq\|y\| \leq \frac{1}{2}+\delta$, próximo de $\|y\|=\frac{1}{2}$. Esta região corresponde a $\rho<\|x\|<\frac{1}{2}$, o primeiro caso de $\psi$ em (4-8). Em particular, vamos examinar os autovetores e autovalores de $\sigma$, correspondentes às condições de condutividade máxima e mínima.

De (4-7) podemos deduzir alguns resultados que tornam a compreensão de $\sigma$, a partir de $\mathbf{D} \phi$, mais interessante. Escreva $\phi(x)=\left(y_{1}, y_{2}\right)$ como definido acima. Agora, pela regra da cadeia, obtemos:

$$
\begin{aligned}
& \nabla y_{1}=\left(\frac{\psi^{\prime}(\|x\|)}{\|x\|^{2}} x_{1}^{2}+\frac{\psi(\|x\|)}{\|x\|^{3}} x_{2}^{2}, \frac{\psi^{\prime}(\|x\|)}{\|x\|^{2}} x_{1} x_{2}-\frac{\psi(\|x\|)}{\|x\|^{3}} x_{1} x_{2}\right) \\
& \nabla y_{2}=\left(\frac{\psi^{\prime}(\|x\|)}{\|x\|^{2}} x_{1} x_{2}-\frac{\psi(\|x\|)}{\|x\|^{3}} x_{1} x_{2}, \frac{\psi^{\prime}(\|x\|)}{\|x\|^{2}} x_{2}^{2}+\frac{\psi(\|x\|)}{\|x\|^{3}} x_{1}^{2}\right)
\end{aligned}
$$

A partir de (4-10) e (4-11), encontramos

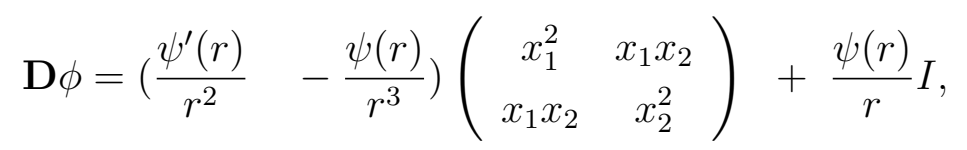

em que $I$ é a matriz identidade e $r=\|x\|=\psi^{-1}(\|y\|)$. Em particular, note que $\mathbf{D} \phi$ é simétrica, de modo que a partir de (4-2) temos $\sigma=\frac{(\mathbf{D} \phi)^{2}}{|\operatorname{det}(\mathbf{D} \phi)|}$. 
As proposições a seguir são úteis para estudarmos os autovetores e autovalores para $\sigma$.

Proposição 4.4.1 Se v é um autovetor com autovalor $\mu$ para uma matriz não singular $A n \times n$, e $B=\frac{A^{2}}{\|\operatorname{det} A\|}$, então v também é um autovetor de $B$, com autovalor $\lambda=\frac{\mu^{2}}{\|\operatorname{det} A\|}$.

Prova: De fato, como $A v=\mu v$, temos que $B v=\frac{A}{\|\operatorname{det} A\|}(A v)=\frac{\mu A v}{\|\operatorname{det} A\|}=$ $\frac{\mu^{2} v}{\|\operatorname{det} A\|}=\lambda v$. Portanto, $v$ é autovetor de $B$ com autovalor $\lambda=\frac{\mu^{2}}{\|\operatorname{det} A\|}$.

Proposição 4.4.2 A matriz $2 \times 2$ com entradas $x_{1}^{2}, x_{1} x_{2}, x_{2}^{2}$ como acima em (4-12) tem autovetores (ortogonais) $\left[x_{1}, x_{2}\right]^{T} e\left[-x_{2}, x_{1}\right]^{T}$, com autovalores $r^{2}=\|x\|^{2}$ e 0 , respectivamente.

Prova: Com efeito, o resultado segue imediatamente através das seguintes verificações:

$$
\begin{aligned}
& \left(\begin{array}{cc}
x_{1}^{2} & x_{1} x_{2} \\
x_{1} x_{2} & x_{2}^{2}
\end{array}\right)\left(\begin{array}{l}
x_{1} \\
x_{2}
\end{array}\right)=\left(\begin{array}{c}
x_{1}^{3}+x_{1} x_{2}^{2} \\
x_{1}^{2} x_{2}+x_{2}^{3}
\end{array}\right)=\left[x_{1}^{2}+x_{2}^{2}\right]\left(\begin{array}{l}
x_{1} \\
x_{2}
\end{array}\right)=\|x\|^{2}\left(\begin{array}{l}
x_{1} \\
x_{2}
\end{array}\right) \\
& \mathrm{e} \\
& \left(\begin{array}{cc}
x_{1}^{2} & x_{1} x_{2} \\
x_{1} x_{2} & x_{2}^{2}
\end{array}\right)\left(\begin{array}{c}
-x_{2} \\
x_{1}
\end{array}\right)=\left(\begin{array}{l}
-x_{1}^{2} x_{2}+x_{1}^{2} x_{2} \\
-x_{1} x_{2}^{2}+x_{1} x_{2}^{2}
\end{array}\right)=\left(\begin{array}{l}
0 \\
0
\end{array}\right)=0\left(\begin{array}{c}
-x_{2} \\
x_{1}
\end{array}\right) .
\end{aligned}
$$

Se podemos calcular os autovetores e autovalores para $\mathbf{D} \phi$, então podemos utilizar a proposição 4.4.1 para encontrar essas quantidades para $\sigma$. Os autovetores e autovalores para $\mathbf{D} \phi$ seguem facilmente da proposição 4.4.2: a multiplicação da matriz com entradas $x_{1}^{2}$, etc, por $\left(\frac{\psi^{\prime}(r)}{r^{2}}-\frac{\psi(r)}{r^{3}}\right)$ e a troca por $\left(\frac{\psi(r)}{r}\right) I$ mostra que $\mathbf{D} \phi$ tem também autovetores $v_{1}=\left[x_{1}, x_{2}\right]^{T}$ e $v_{2}=$ $\left[-x_{2}, x_{1}\right]^{T}$ com autovalores correspondentes $\mu_{1}=\left(\psi^{\prime}(r)-\frac{\psi(r)}{r}\right)+\frac{\psi(r)}{r}=\psi^{\prime}(r)$ e $\mu_{2}=\frac{\psi(r)}{r}$. Note também que $\operatorname{det}(\mathbf{D} \phi)=\mu_{1} \mu_{2}$.

Em seguida, a partir da proposição 4.4.1, descobrimos que $\sigma$ tem autovetores $v_{m}=v_{1}$ e $v_{M}=v_{2}$ (o mesmo que $\mathbf{D} \phi$, mas remarcado para indicar quais serão as direções de máxima e mínima condutividade). Os autovalores correspondentes ou condutividades são: 


$$
\begin{gathered}
\gamma_{m}=\frac{\mu_{1}^{2}}{\mu_{1} \mu_{2}}=\frac{r \psi^{\prime}(r)}{\psi(r)}, \\
\gamma_{M}=\frac{\mu_{2}^{2}}{\mu_{1} \mu_{2}}=\frac{\psi(r)}{r \psi^{\prime}(r)} .
\end{gathered}
$$

Em particular, as condutividades são inversas umas das outras! O vetor $v_{m}$ aponta radialmente para fora da origem e $v_{M}$ é tangente à qualquer círculo centrado na mesma origem. Na verdade, num ponto $x_{1}=r \cos \theta, x_{2}=r \sin \theta$ também podemos ter $v_{m}=(\cos \theta) \widehat{i}+(\sin \theta) \widehat{j}$, desde que os autovetores possam ser rescalados. Similarmente, podemos tomar $v_{M}=(-\sin \theta) \widehat{i}+(\cos \theta) \widehat{j}$. Se usarmos (4-13) para examinar o comportamento de $\sigma$ na região interior da camuflagem $\frac{1}{2}<\|y\|<\frac{1}{2}+\frac{\delta}{2}$ (correspondente a $\rho<\|x\|<\frac{1}{2}$ ) e utilizarmos (4-8), descobrimos que as condutividades desta região são dadas por:

$$
\begin{aligned}
\gamma_{m}(r) & =\frac{2 r \delta}{1+2 r \delta-2 \rho-2 \delta \rho} \\
\gamma_{M}(r) & =\frac{1+2 r \delta-2 \rho-2 \delta \rho}{2 r \delta}=\frac{1}{\gamma_{m}(r)} .
\end{aligned}
$$

Note que podemos expressar os autovalores em termos de $y$ através de $r=$ $\psi^{-1}(\|y\|)$. Na superfície interna $\|y\|=\frac{1}{2}$ (correspondente a $r=\rho$ ) em $\Omega_{1 / 2}$ temos

$$
\gamma_{m}=\frac{2 \rho \delta}{1-2 \rho}, \quad \gamma_{M}=\frac{1-2 \rho}{2 \rho \delta}
$$

Quando $\rho$ tende a zero, $\gamma_{M} \approx \frac{1}{2 \rho \delta}$ é grande, então a condutividade na direção tangencial à circunferência $\|y\|=\frac{1}{2}$ é muito grande. Da mesma forma, $\gamma_{m} \approx 2 \rho \delta$ tende a zero neste caso, então a condutividade na direção normal é baixa. Fisicamente, um "raio" (na verdade, um elétron) se aproxima de $\|y\|=\frac{1}{2}$ e é desviado em direção a alta condutividade, encaminhado tangencialmente em torno da bola $B_{\frac{1}{2}}(0)$, então ejetado para o outro lado para continuar seu caminho. Por exemplo, observe os fluxos perto de $R=\frac{1}{2}$ no gráfico à direita na figura 4.2.

Pode parecer surpreendente que os autovalores para $\sigma$ definidos por (4-2) sejam inversos. Seria uma falha da transformação radial $\phi$ ? Não! A proposição a seguir ratifica esse resultado.

Proposição 4.4.3 Se A é uma matriz $2 \times 2$ não singular, então a matriz $M=\frac{A A^{T}}{|\operatorname{det}(A)|}$ tem autovalores inversos. 
Prova: Com efeito, a matriz $M$ é simétrica e positiva definida. Logo, se $\mu_{1}$ e $\mu_{2}$ são os autovalores de $M$ então $\operatorname{det} M=\mu_{1} \mu_{2}$. Assim,

$$
\mu_{1} \mu_{2}=\operatorname{det} M=\operatorname{det}\left(\frac{A A^{T}}{|\operatorname{det} A|}\right)=\frac{1}{|\operatorname{det} A|^{2}}(\operatorname{det} A)\left(\operatorname{det} A^{T}\right)=\frac{\operatorname{det} t^{2} A}{\operatorname{det}^{2} A}=1 .
$$

Portanto, $\mu_{1} \mu_{2}=1$.

O leitor atento poderia indagar sobre a possibilidade da relação inversa entre os autovalores de $\sigma$ ter surgido da hipótese feita anteriormente com a condutividade constante $\gamma$ unitária e se esta escolha particular não poderia estar "burlando" de alguma forma os resultados. Na verdade, se considerarmos $\gamma>0$ e diferente de 1 os autovalores $\gamma_{M}$ e $\gamma_{m}$, de fato, não serão mais inversos um do outro, porém, ainda serão inversamente proporcionais. Isto é, à luz dos exemplos 3.3.1 e 3.3.2, na região de camuflagem a matriz

$$
\sigma=P\left(\begin{array}{cc}
\gamma_{M} & 0 \\
0 & \gamma_{m}
\end{array}\right) P^{-1}
$$

em que $P$ representa a matriz de autovetores, deve ser semelhante à matriz

$$
\widetilde{\sigma}=\left(\begin{array}{ll}
\gamma & 0 \\
0 & \gamma
\end{array}\right)
$$

Assim, $\gamma_{M} \cdot \gamma_{m}=\operatorname{det}(\sigma)=\operatorname{det}(\widetilde{\sigma})=\gamma^{2}$. Ou seja, $\gamma_{M}=\gamma^{2} \cdot \frac{1}{\gamma_{m}}$. Com efeito, não há perda de generalidade ao considerarmos $\gamma=1$.

Observação 4.4.4 (A camuflagem perfeita)

É natural considerar $\rho \rightarrow 0^{+}$para obter a capa de invisibilidade perfeita. Isso realmente pode ser feito!. No entanto, se observarmos os autovalores para $\sigma$, vemos que de $\sigma$, temos que $\gamma_{m}$ avaliado na fronteira interior $\|y\|=$ $\frac{1}{2}$ tende para zero com $\rho \rightarrow 0$, enquanto $\gamma_{M}$ tende para infinito; ambos autovetores mantêm-se inalterados. Isto corresponde a condutância perfeita ao redor de $\|y\|=\frac{1}{2}$, isolamento perfeito através desta curva, o que pode não ser fisicamente realista. Ainda assim, tornando $\rho$ pequeno, mas diferente de zero, podemos obter um manto "prático" de qualquer força desejada sem comportamento singular.

\section{Observação 4.4.5 (Condutores anisotrópicos e metamateriais)}

Embora muitos materiais naturais têm condutividade anisotrópica, como criar um material com propriedades anisotrópicas desejadas? Uma abordagem consiste em utilizar materiais isotrópicos, homogêneos e introduzir microestrutura periódica, por exemplo, colocar buracos ou rachaduras no material em um 
padrão específico, mas em uma escala muito pequena. Ao impor uma microestrutura periódica obtém-se um material que, macroscopicamente, parece ter propriedades anisotrópicas. A teoria matemática envolvida em analisar como microestruturas periódicas criam certas propriedades macroscópicas é chamada homogeneização e as técnicas se aplicam em muito mais do que condução elétrica; elas podem ser aplicadas a muitas situações que envolvem um sistema físico regido por equações diferenciais.

Não vamos entrar em detalhes sobre a homogeneização aqui, mas como um exemplo, em (5) os autores citam a discussão a respeito da possibilidade de se obter um material condutor que aparece macroscopicamente para ser um condutor elétrico anisotrópico através da introdução de fendas periódicas em um condutor isotrópico homogêneo. Especificamente, considere a caixa $-\varepsilon<x_{1}, x_{2}<\varepsilon$ em $\mathbb{R}^{2}$, com condutividade isotrópica $\gamma$. Introduzimos uma rachadura isolante na caixa; a fenda é linear com centro em (0,0), e encontra-se em ângulo a com relação à horizontal. Os autores mostram que se "azulejarmos" uma região $\Omega$ no plano com uma coleção destas caixas $2 \varepsilon$ por $2 \varepsilon$ e fazendo $\varepsilon \rightarrow 0$, a região $\Omega$ tem condutividade anisotrópica $\sigma$ eficaz de fórmula:

$$
\sigma=\gamma I-\gamma R\left(\begin{array}{cc}
\sin ^{2}(\alpha) & -\sin (\alpha) \cos (\alpha) \\
-\sin (\alpha) \cos (\alpha) & \cos ^{2}(\alpha)
\end{array}\right)
$$

onde $R$ é um parâmetro que depende do ângulo $\alpha$ e do comprimento da fenda em relação à largura da caixa. Ao ajustar o ângulo e comprimento das fissuras (em relação ao seu espaçamento), bem como $\gamma$, pode-se, em princípio, obter qualquer perfil de condutividade anisotrópica. Resultados semelhantes podem ser obtidos através da introdução de orifícios periódicos ou outras formas (Bryan, K.; Vogelius, M., apud, Bryan, K.; Leise, T., 2010). 


\section{5 \\ Conclusão}

É fato que existem atualmente inúmeras técnicas para elaboração de capas ou dispositivos de invisibilidade, com variadas abordagens físicas e matemáticas muitas vezes um tanto complexas. Entretanto, neste trabalho optamos por uma explicação acessível a não especialistas e àqueles que possuem conhecimento restrito em Cálculo Vetorial, Séries de Fourier, Equações Diferenciais e Álgebra Linear.

O objetivo da camuflagem aqui abordado foi o de tornar um objeto invisível à detecção de energia eletromagnética ao seu entorno com um "metamaterial" especialmente projetado que redireciona as ondas eletromagnéticas ao seu redor. Para tanto, mostramos como disfarçar ou camuflar um objeto contra a detecção de tomografia de impedância, uma técnica de processamento de imagem bem recente. Em suma, nossa abordagem para a camuflagem foi fazer com que um círculo grande parecesse pequeno.

Nesse contexto, descrevemos a ideia essencial por trás da abordagem da "Transformação óptica" da camuflagem em duas dimensões para imagem obtida através da tomografia de impedância. Cabe observar que a transformação aqui referenciada é a função $\phi$ da seção 4.1, que determina, por meio de (4-2), as propriedades necessárias à condutividade de camuflagem. Optamos em expor essas informações no âmbito bidimensional por simples conveniência. Não obstante, de forma a tornar-se mais realista, essas ideias ainda podem ser aplicadas às equações de Maxwell em três dimensões (para uma visão geral dessas equações, veja pp. 358-361 de [19]), em frequências não nulas, e utilizando uma mudança singular de variáveis a fim de alcançar uma camuflagem perfeita ao invés de uma quase-camuflagem.

Uma pergunta chave a ser feita é acerca da possibilidade de camuflar sobre uma grande faixa de frequências, ao invés de meramente em uma frequência particular, pois a faixa de frequência pode ser severamente restrita para algumas formulações de camuflagem. Contudo, os problemas dessa natureza são primeiramente físicos e de engenharia, não matemáticos. Ao se evitar os metamateriais, cujas propriedades dependem da ressonância, recentemente, pesquisadores descobriram que a camuflagem para uma série de frequências no 
espectro eletromagnético pode ser de fato possível e até capaz de funcionar para a luz visível.

O campo da camuflagem tem se mostrado extremamente ativo, com muitas ideias interessantes surgindo. Por exemplo, em 2009 cientistas apresentaram um dispositivo que encobre um objeto à distância (o dispositivo é projetado especialmente para um determinado objeto em uma distância específica em relação ao dispositivo de camuflagem). Também pode-se gerar efeitos de camuflagem através da ressonância anômala localizada, que ocorre perto de uma "superlente", um metamaterial com índice de refração negativo que produz resolução mais fina do que o comprimento de onda da luz a ser utilizado a fim de gerar a imagem.

O tema da camuflagem tem sido também explorado em outros contextos além de ondas eletromagnéticas, como por exemplo ondas de elasticidade e ondas de matéria (camuflagem quantum). O estudo nessa temática deve seguir o mesmo caminho que toda pesquisa bem sucedida e que alcançou aplicabilidades no mundo real trilhara: teve sua infância, possivelmente está passando pela adolescência, e, provavelmente, chegará à fase adulta. Conta a lenda que, no século XIX, quando Michael Faraday fazia uma demonstração sobre sua mais nova descoberta, a indução eletromagnética, foi perguntado por um dos presentes: "Mas, para que serve tudo isso ?". Faraday, então, prontamente respondeu: "Para que serve uma criança recém nascida ?".

$\mathrm{O}$ assunto da camuflagem sugere muitos projetos de pesquisa interessantes para serem investigados. Listamos a seguir algumas sugestões abertas para possíveis direções de pesquisa. Cabe ressaltar que nenhuma reivindicação é feita ou implícita sobre a facilidade ou até mesmo a possibilidade de resolvelas. Em particular, a área está evoluindo rapidamente, com muitas pessoas trabalhando em ideias relacionadas ao primeiro projeto a seguir:

1. Nossa abordagem para camuflagem era fazer um buraco grande parecer um pequeno buraco. Podemos fazer o inverso, um pequeno buraco parecer grande? Mesmo mais genericamente, pode esta técnica de mudança de variáveis ser usada para disfarçar, em vez de esconder $D$ ? Por exemplo, pode $D=B_{\frac{1}{2}}(0)$ se fazer parecer como uma elipse ou alguma outra forma? Quais são as limitações?

2. Seria possível construir uma "camuflagem direcional" que torna um objeto (aproximadamente) invisível de algumas direções, totalmente visíveis dos outros? Pense em algum tipo de dispositivo que você carregue para a batalha, para que da frente (onde seus inimigos estão) você está 
invisível, mas por trás (onde seus aliados estão) você está visível. (Este conceito é baseado em uma pergunta feita por J. Christopher Tweddle na Universidade de Evansville)

3. Outra forma de energia que tem sido utilizado para a imagem é o calor. Suponha que $v\left(x_{1}, x_{2}, t\right)$ satisfaça a equação do calor $v_{t}-\Delta u=0$ no disco unitário $\Omega=B_{1}(0)$ (aqui $v$ é a temperatura de $\Omega$ ). Para simplificar, suponha que $v$ é tempo-harmônica, isto é, $v\left(x_{1}, x_{2}, t\right)=e^{\imath \omega t} u\left(x_{1}, x_{2}\right)$. Então $\Delta u+\imath \omega u=0$. Um observador examina o interior de $\Omega$ impondo uma temperatura $u=f$ em $\partial \Omega$, então mede o fluxo de calor $\frac{\partial u}{\partial n}$ em $\partial \Omega$. Podemos encobrir um vazio $D=B_{\frac{1}{2}}(0)$, utilizando a técnica para obtenção de imagem por impedância? Se $\omega=0$ é o mesmo problema, então supomos que $\omega>0$. 


\section{Referências Bibliográficas}

[1] A. GREenleAF, Y. KURYLEV, M. L.; UhlmAnN, G.. Cloaking devices, electromagnetic wormholes, and transformation optics. SIAM Rev., 51(2009):pp. 3-33.

[2] ABRAhAM, R.; MARSDEN, J. E.; RATIU, T. S. ; CUSHMAN, R.. Foundations of mechanics. Benjamin/Cummings Publishing Company, 1978.

[3] BARTLE, R. G.; BARTLE, R. G.. The elements of integration and Lebesgue measure. Wiley Online Library, 1995.

[4] BREZIS, H.. Analisi funzionale. Liguori Editore Srl, 1986.

[5] BRYAN, K.; LEISE, T.. Impedance imaging, inverse problems, and harry potter's cloak. SIAM Review, 52(2):359-377, 2010.

[6] COURANT, R.; HILBERT, D.. Methods of Mathematical Physics: Volume II, volumen 2. WILEY, 1989.

[7] EVANS, L.. Partial differential equations. American Mathematical Society Providence, RI, 1998.

[8] FOLLAND, G. B.; FOLLAND, G.. Real analysis: modern techniques and their applications, volumen 2. Wiley New York, 1984.

[9] GILBARG, D.; TRUDINGER, N.. Elliptic Partial Differential Equations of Second Order. Springer, 2001.

[10] KELLER, J. B.. Inverse problems. Mathematical Association of America, 83(2, Feb. 1976):pp. 107-118.

[11] KRANTZ, S. G.; PARKS, H. R.. Geometric integration theory. Birkhäuser Boston, 2008.

[12] KREYSZIG, E.. Introductory functional analysis with applications. Wiley New York, 1978. 
[13] LIMA, E. L.. Algebra linear, sétima ediçao. SBM, Coleçao Matemática Universitária, 2004.

[14] LIMA, E. L.. Curso de análise vol. 2 (oitava ediçao). Projeto Euclides. IMPA, 2005.

[15] MAUREY, B.; MILMAN, V. ; TOMCZAK-JAEGERMANN, N.. Asymptotic infinite-dimensional theory of banach spaces. In: GEOMETRIC ASPECTS OF FUNCTIONAL ANALYSIS, p. 149-175. Springer, 1995.

[16] MUNKRES, J. R.. Topology. Prentice Hall, 2nd edition, 2000.

[17] PAVliOTIS, G. A.; STUART, A.. Multiscale Methods Averaging and Homogenization. Springer, 2010.

[18] ROYDEN, H.. Real analysis. Macmillan New York, 1968.

[19] STRAUSS, W. A.. Partial Differential Equation: An Introduction. John Wiley, New York, 1992.

[20] TREFETHEN, L. N.; BAU III, D.. Numerical linear algebra. Society for Industrial Mathematics, 1997. 


\section{A \\ Alguns resultados pertinentes}

\section{A.1}

\section{Impedância elétrica e formas diferenciais das equações de Maxwell}

A impedância elétrica $Z$ é uma grandeza normalmente usada em circuitos elétricos e mede a razão entre a voltagem efetiva $V_{\text {ef }}$ aplicada no circuito e a corrente elétrica efetiva $I_{e f}$ que o atravessa:

$$
Z=\frac{V_{e f}}{I_{e f}}
$$

Conceitualmente, a impedância elétrica mede a oposição total que um dado circuito apresenta à passagem da corrente elétrica. Para um circuito resistivo e capacitivo de corrente alternada, ela também pode ser expressa como o inverso da soma de dois termos, uma parte real representando o termo resistivo e uma parte complexa representando o termo capacitivo:

$$
\frac{1}{Z}=\sigma(x, \omega)+\omega \varepsilon(x, \omega) \imath
$$

em que $x$ representa a posição, $\sigma$ a condutividade elétrica, $\varepsilon$ a permissividade elétrica (propriedade elétrica que mede a facilidade com que as cargas elétricas do material se separam sob a ação de um campo elétrico) e $\omega$ a frequência angular da corrente elétrica $(\omega=2 \pi f$-onde $f$ é a frequência em $\mathrm{Hz})$.

Na Tomografia de Impedância Elétrica é possível construir uma imagem do objeto a partir da sua condutividade $\sigma$ ou da sua permissividade $\varepsilon$. No entanto, consideraremos o meio puramente condutivo de forma que possamos desprezar os efeitos capacitivos, e, consequentemente, o termo de permissividade elétrica. Dessa forma, o imageamento é feito exclusivamente pela condutividade elétrica do objeto.

A formulação matemática da Tomografia de Impedância Elétrica corresponde, essencialmente, a EDP que rege o potencial elétrico no interior de $\Omega$ e as condições de fronteira adequadas que representam o processo de "estímulo- 
resposta". A dedução da EDP se faz a partir das Equações de Maxwel

$$
\begin{aligned}
\nabla \cdot \mathbf{E} & =\frac{\widehat{\rho}}{\varepsilon} \\
\nabla \times \mathbf{E} & =-\frac{\partial \mathbf{B}}{\partial t} \\
\nabla \cdot \mathbf{B} & =0 \\
\nabla \times \mathbf{B} & =\mu \mathbf{J}-\mu \varepsilon \frac{\partial \mathbf{E}}{\partial t}
\end{aligned}
$$

em que $\mathbf{E}$ é o campo elétrico, $\mathbf{B}$ o campo magnético, $\mathbf{J}$ é a densidade de corrente elétrica, $\widehat{\rho}$ é a densidade de carga elétrica, $\varepsilon$ é a permissividade elétrica e $\mu$ é a permeabilidade magnética. Para diminuir a complexidade do modelo, podemos fazer algumas aproximações. Uma delas é desprezar os possíveis efeitos da indução eletromagnética, uma vez que podemos aplicar frequencias de correntes ou tensões relativamente baixas. Assim, podemos igualar a zero todas as derivadas em relação ao tempo das equações de Maxwel. Além disso, outra simplificação a considerar no problema de tomografia de impedância elétrica é a inexistência de fontes internas de campo magnético. Com isso, as equações resumem-se na relação entre o campo elétrico $\mathbf{E}$ e a densidade de carga elétrica $\widehat{\rho}$

$$
\nabla \cdot \mathbf{E}=\frac{\widehat{\rho}}{\varepsilon}
$$

Por sua vez, pelo fato de $\mathbf{E}=-\nabla u$, a lei de Ohm em (3-1), para condutividade $\sigma$, e por considerar que não há fontes internas de corrente $(\log o, \widehat{\rho}=0)$, concluímos que a equação $\nabla(-\sigma \nabla u)=0$ em $\Omega$ é a equação principal no problema da tomografia de impedância elétrica. Como vimos anteriormente, esta EDP rege o potencial elétrico $u\left(x_{1}, x_{2}\right)$ no interior de $\Omega$, sendo $\sigma\left(x_{1}, x_{2}\right)$ a condutividade elétrica dos pontos internos do domínio.

\section{A.2}

\section{Teorema da Divergência e as Identidades de Green}

Embora os resultados dessa seção sejam válidos em $\mathbb{R}^{n}$, estudaremos apenas o caso $n=2$. Inicialmente enunciaremos o Teorema de Green no plano e demonstraremos o Teorema da Divergência. Ainda nessa seção, descreveremos algumas fórmulas, as chamadas identidades de Green, que são muito úteis em EDP. Demonstraremos essas identidades em $\mathbb{R}^{2}$.

Um teorema fundamental para o estudo das EDP's lineares é o Teorema da Divergência. Como o Teorema da Divergência em $\mathbb{R}^{2}$ é um corolário do Teorema de Green, vamos lembrar, do curso de cálculo, o enunciado deste teorema. (para mais informações, veja [19]. 
Teorema A.2.1 (Teorema de Green) Seja $\Omega \subset \mathbb{R}^{2}$ aberto, conexo e limitado, e sejam $P, Q: \bar{\Omega} \rightarrow \mathbb{R}$ funções contínuas. Suponha que:

1. $\partial \Omega$ é uma curva fechada simples (isto é, sem auto-interseções) retificável;

2. as derivadas parciais $Q_{x}$ e $P_{y}$ existem e são limitadas em $\Omega$;

3. as integrais duplas $\int_{\bar{\Omega}} Q_{x} d x d y, \int_{\bar{\Omega}} P_{y} d x d y$ existem.

Então a integral de linha $\int_{\partial \Omega^{+}}(P d x+Q d y)$ existe $e$

$$
\int_{\bar{\Omega}}\left(Q_{x}-P_{y}\right) d x d y=\int_{\partial \Omega^{+}}(P d x+Q d y) .
$$

Intuitivamente, a orientação positiva de $\partial \Omega$ é tal que andando em cima de $\partial \Omega$ a região $\Omega$ está sempre a nossa esquerda.

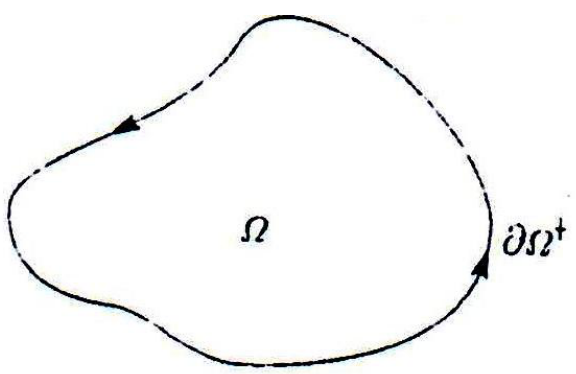

Figura A.1: Orientação positiva de $\partial \Omega$.

O teorema de Green, como enunciado acima, supõe que o domínio $\Omega$ seja simplesmente conexo (isto é, sem "buracos"). No caso em que $\Omega$ tem um número finito de "buracos", podemos reduzir ao caso do teorema A.2.1 usando curvas auxiliares como na Figura A.2: a integral sobre $\Omega$ se escreve como uma soma finita de integrais sobre regiões sem "buracos".

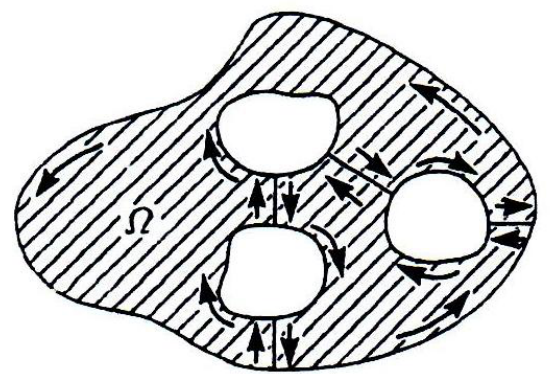

Figura A.2: O teorema de Green também é válido para domínios multiplamente conexos.

Antes de enunciarmos o teorema da divergência, vamos introduzir a noção de normal externa. Suponha que $\Omega$ é aberto, conexo e limitado, tal 
que $\partial \Omega$ é uma curva suave, isto é, admite uma parametrização continuamente diferenciável $\alpha$ tal que o vetor velocidade $\alpha^{\prime}(t)$ nunca se anula. Mergulhe $\Omega$ em $\mathbb{R}^{3}$ considerando, por exemplo, $\Omega$ contido no plano $z=0$ e defina

$$
\widehat{n}(t)=\frac{\alpha^{\prime}(t) \times \vec{k}}{\left|\alpha^{\prime}(t) \times \vec{k}\right|}
$$

onde $\vec{k}=(0,0,1)$ e $\times$ é o produto vetorial em $\mathbb{R}^{3}$. Se a parametrização $\alpha$ dá uma orientação positiva a $\partial \Omega, \widehat{n}$ definida como em (A-6) é a normal externa unitária (veja a Figura A.3 - na figura, o vetor $\vec{k}$ "sai" do papel). Calculando explicitamente o produto vetorial em (A-6) se $\alpha(t)=(x(t), y(t))$,

$$
\alpha^{\prime}(t) \times \vec{k}=\left|\begin{array}{ccc}
\vec{i} & \vec{j} & \vec{k} \\
x^{\prime}(t) & y^{\prime}(t) & 0 \\
0 & 0 & 1
\end{array}\right|=\left(y^{\prime}(t),-x^{\prime}(t), 0\right)
$$

Voltando para $\mathbb{R}^{2}$, a fórmula (A-6) fica:

$$
\widehat{n}(t)=\frac{1}{\sqrt{\left(x^{\prime}(t)\right)^{2}+\left(y^{\prime}(t)\right)^{2}}}\left(y^{\prime}(t),-x^{\prime}(t)\right)
$$

Vamos convencionar que, sempre que falamos em normal unitária à curva $\alpha$ no ponto $t$, estaremos nos referindo a $\widehat{n}(t)$ definida por (A-7). Como observamos acima, no caso de curvas fechadas orientadas positivamente, $\widehat{n}$ é a normal externa unitária. Note também que podemos definir $\widehat{n}$ por (A-7) a menos de um número finito de pontos se $\alpha$ for uma união finita de curvas suaves.

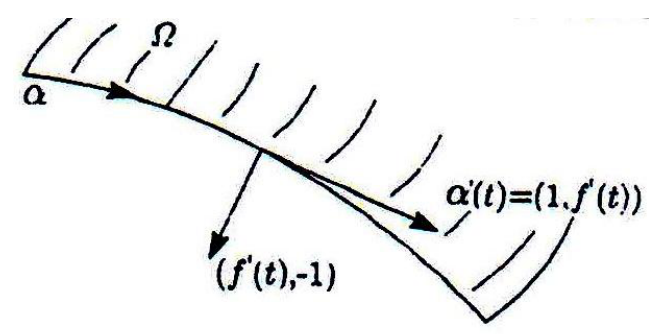

Figura A.3: Se a parametrização $\alpha$ dá uma orientação positiva a $\partial \Omega, \widehat{n}(t)$ é a normal externa unitária no ponto $\alpha(t)$.

Uma curva suave pode ser sempre parametrizada pelo comprimento de arco pois $\frac{d s}{d t}=\left|\alpha^{\prime}(t)\right|>0$. Em particular, se $f: U \subset \mathbb{R}^{2} \rightarrow \mathbb{R}$ é uma função seccionalmente contínua e $\alpha:[a, b] \rightarrow U$ é uma curva suave, então a integral de $f$ ao longo de $\alpha$ em relação ao comprimento de arco é $\int_{\alpha} f d s=\int_{a}^{b} f(\alpha(t))\left|\alpha^{\prime}(t)\right| d t=\int_{0}^{l} f(\beta(s)) d s$, onde $l$ é o comprimento de $\alpha$ e 
$\beta: s \in[0, l] \mapsto \alpha(t(s)) \in U$ é a reparametrização de $\alpha$ pelo comprimento de arco.

Teorema A.2.2 (Teorema da Divergência) Seja $\Omega \subset \mathbb{R}^{2}$ aberto, conexo e limitado, cuja fronteira $\partial \Omega$ é uma união finita de curvas suaves. Seja $F: \bar{\Omega} \rightarrow$ $\mathbb{R}^{2}$ um campo vetorial de classe $C^{1}$ em $\bar{\Omega}$. Então

$$
\int_{\bar{\Omega}} \nabla \cdot F d x d y=\int_{\partial \Omega} F \cdot \widehat{n} d s
$$

onde $\widehat{n}$ é a normal externa.

Prova: Sejam $\alpha_{1}, \ldots, \alpha_{k}$ as curvas que compõem $\partial \Omega$, onde $\alpha_{i}: t \in\left[a_{i}, b_{i}\right] \mapsto$ $\left(x_{i}(t), y_{i}(t)\right) \in \mathbb{R}^{2}$ dão uma orientação positiva a $\partial \Omega, i=1, \ldots, k$. Se $\Omega$ for simplesmente conexo, $\partial \Omega$ é uma curva simples fechada seccionalmente $C^{1}$, logo retificável e portanto o Teorema de Green é válido em $\Omega$. Se $\Omega$ não for simplesmente conexo, como $\partial \Omega$ é composto por um número finito de curvas suaves, $\Omega$ tem um número finito de "buracos" e portanto, usando curvas auxiliares (veja figura 34), $\Omega$ pode ser decomposto em um número finito de subconjunto abertos, conexos e limitados (e partes de suas fronteiras, o que não altera a integral dupla) que satisfazem as condições do teorema A.2.1, logo o teorema de Green é válido em $\Omega$. Portanto, se $F=\left(F_{1}, F_{2}\right)$, tomando $P=-F_{2}$ e $Q=F_{1}$, podemos aplicar o teorema de Green para obter

$$
\begin{aligned}
\int_{\bar{\Omega}} \nabla \cdot F d x d y & =\int_{\bar{\Omega}}\left(\partial_{x} Q-\partial_{y} P\right) d x d y \\
& =\int_{\partial \Omega^{+}}(P d x+Q d y) \\
& =\sum_{i=1}^{k} \int_{\alpha_{i}}(P d x+Q d y) \\
& =\sum_{i=1}^{k} \int_{a_{i}}^{b_{i}}\left[-F_{2}\left(\alpha_{i}(t)\right) x_{i}^{\prime}(t)+F_{1}\left(\alpha_{i}(t)\right) y_{i}^{\prime}(t)\right] d t \\
& =\sum_{i=1}^{k} \int_{a_{i}}^{b_{i}}\left(F_{1}\left(\alpha_{i}(t)\right), F_{2}\left(\alpha_{i}(t)\right)\right) \cdot\left(y_{i}^{\prime}(t),-x_{i}^{\prime}(t)\right) d t \\
& =\sum_{i=1}^{k} \int_{a_{i}}^{b_{i}} F\left(\alpha_{i}(t)\right) \cdot \widehat{n}_{i}(t)\left|\alpha_{i}^{\prime}(t)\right| d t \\
& =\sum_{i=1}^{k} \int_{0}^{l\left(\alpha_{i}\right)} F \cdot \widehat{n}_{i} d s=\int_{\partial \Omega} F \cdot \widehat{n} d s
\end{aligned}
$$

Teorema A.2.3 Seja $\Omega \subset \mathbb{R}^{2}$ aberto e conexo em que vale o teorema da divergência e sejam $u, v \in C^{2}(\bar{\Omega})$. Então valem as seguintes identidades: 


$$
\begin{gathered}
\int_{\bar{\Omega}}(v \Delta u+\nabla v \cdot \nabla u) d x d y=\int_{\partial \Omega} v \frac{\partial u}{\partial n} d s \\
\int_{\bar{\Omega}}(v \Delta u-u \Delta v) d x d y=\int_{\partial \Omega}\left(v \frac{\partial u}{\partial n}-u \frac{\partial v}{\partial n}\right) d s
\end{gathered}
$$

onde $\frac{\partial}{\partial n}$ é a derivada direcional na direção da normal unitária externa $\widehat{n}$.

Prova: Para demonstrar (A-9), seja $F=v \nabla u$. Então $F$ é um campo vetorial de classe $C^{1}$ em $\bar{\Omega}$ e

$$
\nabla \cdot F=\nabla \cdot(v \nabla u)=\nabla v \cdot \nabla u+v \Delta u
$$

Aplicando o teorema da divergência, obtemos

$$
\begin{aligned}
\int_{\bar{\Omega}}(\nabla v \cdot \nabla u+v \Delta u) d x d y & =\int_{\bar{\Omega}} \nabla \cdot F d x d y \\
& =\int_{\partial \Omega} F \cdot \widehat{n} d s=\int_{\partial \Omega} v \nabla u \cdot \widehat{n} d s=\int_{\partial \Omega} v \frac{\partial u}{\partial n} d s .
\end{aligned}
$$

Agora, para provar (A-10) basta usar (A-9):

$$
\begin{aligned}
\int_{\bar{\Omega}}(v \Delta u-u \Delta v) d x d y & =\int_{\bar{\Omega}}(v \Delta u+\nabla v \cdot \nabla u) d x d y-\int_{\bar{\Omega}}(u \Delta v+\nabla u \cdot \nabla v) d x d y \\
& =\int_{\partial \Omega} v \frac{\partial u}{\partial n} d s-\int_{\partial \Omega} u \frac{\partial v}{\partial n} d s
\end{aligned}
$$

A fórmula (A-9) é conhecida como a primeira identidade de Green, enquanto que (A-10) é a segunda identidade de Green.

Corolário A.2.4 Sejam $\Omega \subset \mathbb{R}^{2}$ aberto e conexo em que vale o teorema da divergência e $u \in C^{2}(\bar{\Omega})$ tal que $\Delta u=0 \mathrm{em} \Omega$. Então valem as seguintes identidades:

$$
\begin{gathered}
\int_{\bar{\Omega}}|\nabla u|^{2} d x d y=\int_{\partial \Omega} u \frac{\partial u}{\partial n} d s, \\
\int_{\partial \Omega} \frac{\partial u}{\partial n} d s=0
\end{gathered}
$$

Prova: Para provar (A-11) basta tomar $u=v$ em (A-9); e quanto a (A-12), basta tomar $v \equiv 1$ em (A-10).

Corolário A.2.5 Sejam $\Omega \subset \mathbb{R}^{2}$ aberto e conexo em que vale o teorema da divergência, $f \in C(\partial \Omega)$ e $g \in C^{2}(\partial \Omega)$. Então o problema

$$
\begin{aligned}
u & \in C^{2}(\bar{\Omega}), \\
\Delta u & =f \quad \text { em } \quad \Omega, \\
u & =g \text { em } \quad \partial \Omega
\end{aligned}
$$


tem no máximo uma solução.

Prova: Se $u$ e $v$ são soluções de (A-13), seja $w=u-v$. Então $w$ é solução de

$$
\begin{array}{rlll}
w & \in C^{2}(\bar{\Omega}), \\
\Delta w=0 & \text { em } \quad \Omega, \\
w=0 & \text { em } \quad \partial \Omega .
\end{array}
$$

Como $w=0$ em $\partial \Omega$, de (A-11) temos $\int_{\bar{\Omega}}|\nabla w|^{2} d x d y=0$ e, portanto, como $w \in C^{2}(\bar{\Omega}), \nabla w \equiv 0$ em $\bar{\Omega}, \log$ o é constante em $\bar{\Omega}$ (uma vez que $\Omega$ é conexo) e $w \equiv 0$ (pois $w=0$ em $\partial \Omega$ ).

Nosso próximo objetivo é provar a chamada terceira identidade de Green. Para simplificar a notação, denotaremos os pontos em $\mathbb{R}^{2}$ por letras gregas $\xi$, $\eta$, etc. Por exemplo: $U \subset \mathbb{R}^{2}$ aberto e limitado, e $f: \bar{U} \rightarrow \mathbb{R}$ integrável em $\bar{U}$, escrevemos

$$
\int_{\bar{U}} f(x, y) d x d y:=\int_{\bar{U}} f(\xi) d \xi
$$

A função $F: \mathbb{R}^{2} \backslash\{0\} \rightarrow \mathbb{R}$ definida por

$$
F(\xi)=\frac{1}{2 \pi} \ln |\xi|, \quad \xi \neq 0
$$

é dita solução fundamental de $\Delta u=0 \mathrm{em} \mathbb{R}^{2}$.

Seja $F$ a solução fundamental de $\Delta u=0$ em $\mathbb{R}^{2}$. Para cada $\xi \in \mathbb{R}^{2}$, denotamos por $F_{\xi}$ a função $F_{\xi}(\eta)=F(\xi-\eta)$ definida para $\eta \neq \xi$. Com essa notação, vejamos alguns resultados:

Lema A.2.6 Sejam $\xi \in \mathbb{R}^{2}, R>0$ e $g: \overline{B(\xi ; R)} \rightarrow \mathbb{R}$. Se g é limitada e $F_{\xi} g$ é integrável em relação ao comprimento de arco ao longo de qualquer círculo centrado em $\xi$ de raio $r \in(0, R)$, então

$$
\lim _{r \rightarrow 0^{+}} \int_{\partial B(\xi, r)} F_{\xi} g d s=0 .
$$

Prova: Seja $M>0$ tal que $|g(\eta)| \leq M, \forall \eta \in \overline{B(\xi ; R)}$. Então, se $0<r<R$,

$$
\begin{aligned}
\int_{\partial B(\xi ; r)} F_{\xi} g d s & =\int_{|\xi-\eta|=r} F(\xi-\eta) g(\eta) d s(\eta) \\
& =\frac{\ln r}{2 \pi} \int_{|\xi-\eta|=r} g(\eta) d s(\eta) \\
& \Rightarrow\left|\int_{\partial B(\xi ; r)} F_{\xi} g d s\right| \leq M \frac{|\ln r|}{2 \pi} \int_{|\xi-\eta|=r} d s(\eta)=M r|\ln r| \rightarrow 0
\end{aligned}
$$


quando $r \rightarrow 0^{+}$.

Lema A.2.7 Sejam $\xi \in \mathbb{R}^{2}, R>0$ e $g \in C(\overline{B(\xi ; R)})$. Então

$$
\lim _{r \rightarrow 0^{+}} \int_{\partial B(\xi ; r)} \frac{\partial F_{\xi}}{\partial n} g d s=g(\xi)
$$

em que $\frac{\partial}{\partial n}$ denota a derivada direcional na direção da normal unitária externa $\widehat{n}$.

Prova: Se $\xi=(a, b)$ e $\eta=(x, y)$, então:

$$
\begin{aligned}
F_{\xi}(\eta) & =\frac{1}{2 \pi} \ln |\xi-\eta|=\frac{1}{2 \pi} \ln \sqrt{(x-a)^{2}+(y-b)^{2}}, \\
\partial_{x} F_{\xi}(\eta) & =\frac{1}{2 \pi} \frac{x-a}{|\xi-\eta|^{2}} \\
\partial_{y} F_{\xi}(\eta) & =\frac{1}{2 \pi} \frac{y-b}{|\xi-\eta|^{2}} .
\end{aligned}
$$

Além disso, se $\eta \in \partial B(\xi ; r)$, para $r \in(0, R),|\xi-\eta|=r$ e $\widehat{n}(\eta)=\frac{\eta-\xi}{|\eta-\xi|}$, então

$$
\begin{aligned}
\int_{\partial B(\xi ; r)} \frac{\partial F_{\xi}}{\partial n} g d s & =\int_{|\xi-\eta|=r} \nabla F_{\xi}(\eta) \cdot \widehat{n}(\eta) g(\eta) d s(\eta) \\
& =\frac{1}{2 \pi} \int_{|\xi-\eta|=r} \frac{\eta-\xi}{|\xi-\eta|^{2}} \cdot \frac{\eta-\xi}{|\xi-\eta|} g(\eta) d s(\eta) \\
& =\frac{1}{2 \pi r} \int_{|\xi-\eta|=r} g(\eta) d s(\eta)
\end{aligned}
$$

que é o valor médio de $g$ sobre o círculo de raio $r$. Queremos mostrar, então, que o valor médio de $g$ sobre o círculo de raio $r$ centrado em $\xi$ tende ao valor de $g$ no centro, $g(\xi)$, quando $r \rightarrow 0^{+}$. De fato, como $g$ é contínua em $\overline{B(\xi ; R)}$, $g$ é uniformemente contínua, $\operatorname{logo}$, dado $\epsilon>0$, existe $\delta>0$ tal que

$$
\eta, \eta^{\prime} \in \overline{B(\xi ; R)},\left|\eta-\eta^{\prime}\right|<\delta \Rightarrow\left|g(\eta)-g\left(\eta^{\prime}\right)\right|<\epsilon
$$

então, se $0<r<\delta$,

$$
\begin{aligned}
\left|\int_{\partial B(\xi ; r)} \frac{\partial F_{\xi}}{\partial n} g d s-g(\xi)\right| & =\left|\frac{1}{2 \pi r} \int_{|\xi-\eta|=r}(g(\eta)-g(\xi)) d s(\eta)\right| \\
& \leq \frac{1}{2 \pi r} \int_{|\xi-\eta|=r}|g(\eta)-g(\xi)| d s(\eta)<\epsilon,
\end{aligned}
$$

o que prova $(\mathrm{A}-16)$. 
Lema A.2.8 Dado $\xi \in \Omega$, seja $R>0$ tal que $B(\xi ; R) \subset \Omega$. Para cada $r \in(0, R)$, seja $\Omega_{r}=\Omega-\overline{B(\xi ; r)}$. Então, qualquer que seja $g \in C(\bar{\Omega})$, existe o limite

$$
\lim _{r \rightarrow 0^{+}} \int_{\bar{\Omega}_{r}} F_{\xi}(\eta) g(\eta) d \eta
$$

Prova: Seja

$$
I(r)=\int_{\bar{\Omega}_{r}} F_{\xi}(\eta) g(\eta) d \eta
$$

É claro que a integral em (A-18) existe se $0<r<R$, pois $F_{\xi} g \in C\left(\bar{\Omega}_{r}\right)$. Como $g \in C(\bar{\Omega})$ e $\bar{\Omega}$ é compacto, $g$ é limitada, logo existe $M>0$ tal que $|g(\eta)| \leq M, \forall \eta \in \bar{\Omega}$. Sejam $0<s<t<R$, então

$$
\begin{aligned}
|I(t)-I(s)| & =\left|\int_{\bar{\Omega}_{t}-\bar{\Omega}_{s}} F_{\xi}(\eta) g(\eta) d \eta\right| \\
& \leq \frac{M}{2 \pi} \int_{s<|\xi-\eta| \leq t}|\ln | \xi-\eta|| d \eta .
\end{aligned}
$$

Para calcular essa última integral, introduzimos um sistema de coordenadas polares centrado em $\xi$ (ver Figura A.4): $\eta=\xi+(r \cos \theta, r \sin \theta), s<r \leq t$, $0 \leq \theta \leq 2 \pi$. Então

$$
\int_{s<|\xi-\eta| \leq t}|\ln | \xi-\eta|| d \eta=\int_{s}^{t} r d r \int_{0}^{2 \pi} d \theta|\ln r|=2 \pi \int_{s}^{t} r|\ln r| d r
$$

Por outro lado, $\lim _{r \rightarrow 0^{+}}(r \ln r)=0$, logo a função $r \ln r$ é limitada perto de zero, isto é, existe $N \stackrel{r \rightarrow 0}{>}$ tal que $0<r<1 \Rightarrow 0<r \ln r<N$. Dado $\epsilon>0$ tome $\delta=\frac{\epsilon}{M N}$ : se $\left\{r_{n}\right\} \subset(0, R)$ é tal que $r_{n} \rightarrow 0$ quando $n \rightarrow+\infty$, então existe $n_{0} \in \mathbb{N}$ tal que $n, m \geq n_{0} \Rightarrow\left|r_{n}-r_{m}\right|<\delta \Rightarrow\left|I\left(r_{n}\right)-I\left(r_{m}\right)\right| \leq M N\left|r_{n}-r_{m}\right|<\epsilon$ e portanto, pelo critério de Cauchy, existe $\lim _{n \rightarrow+\infty} I\left(r_{n}\right)$. Como $I\left(r_{n}\right)$ tem limite qualquer que seja a sequência $\left\{r_{n}\right\} \subset(0, R)$ convergindo a zero, existe o limite de $I(r)$ quando $r \rightarrow 0^{+}$.

Agora estamos prontos para abordar a terceira identidade de Green:

Teorema A.2.9 (Terceira Identidade de Green) Seja $\Omega \subset \mathbb{R}^{2}$ aberto e conexo em que vale o teorema da divergência e seja $u \in C^{2}(\bar{\Omega})$. Então, qualquer que seja $\xi \in \Omega$,

$$
u(\xi)=\int_{\partial \Omega}\left(u \frac{\partial F_{\xi}}{\partial n}-\frac{\partial u}{\partial n} F_{\xi}\right) d s+\int_{\bar{\Omega}} F_{\xi}(\eta) \Delta u(\eta) d \eta
$$


Prova: Inicialmente, fixemos $\xi \in \Omega$ e tomemos $R>0$ tal que $B(\xi ; R) \subset \Omega$. Como no lema (A.2.8), se $0<r<R, \Omega_{r}=\Omega-\overline{B(\xi ; r)}$. Observe que $\partial \Omega_{r}=\partial \Omega \cup \partial B(\xi ; r)$ e que a orientação positiva de $\partial \Omega_{r}$ coincide com a orientação positiva de $\partial \Omega$ e com a orientação negativa de $\partial B(\xi ; r)$ (veja Figura A.5); em outras palavras, se $\widehat{n}$ é a normal externa unitária a $\partial \Omega_{r}$ e $\widetilde{n}$ é a normal externa unitária a $\partial B(\xi ; r)$, então $\widetilde{n}=-\widehat{n}$ em $\partial B(\xi ; r)$. Usando o fato que $\Delta F_{\xi}(\eta)=0$ se $\eta \in \bar{\Omega}_{r}$ e aplicando a segunda identidade de Green com $v=F_{\xi}$, obtemos:

$$
\begin{aligned}
\int_{\bar{\Omega}_{r}} F_{\xi} \Delta u d \eta & =\int_{\bar{\Omega}_{r}}\left(F_{\xi} \Delta u-u \Delta F_{\xi}\right) d \eta \\
& =\int_{\partial \Omega_{r}}\left(F_{\xi} \frac{\partial u}{\partial n}-u \frac{\partial F_{\xi}}{\partial n}\right) d s \\
& =\int_{\partial \Omega}\left(F_{\xi} \frac{\partial u}{\partial n}-u \frac{\partial F_{\xi}}{\partial n}\right) d s-\int_{\partial B(\xi ; r)}\left(F_{\xi} \frac{\partial u}{\partial \widetilde{n}}-u \frac{\partial F_{\xi}}{\partial \widetilde{n}}\right) d s .
\end{aligned}
$$

$\underset{0}{0} \quad$ Como $\bar{\Omega}$ é compacto e $\nabla u \cdot \widetilde{n}=\frac{\partial u}{\partial \widetilde{n}}$ é contínua em $\bar{\Omega}, \frac{\partial u}{\partial \widetilde{n}}$ é limitada, logo, pelo lema A.2.6,

$$
\lim _{r \rightarrow 0^{+}} \int_{\partial B(\xi ; r)} F_{\xi} \frac{\partial u}{\partial \widetilde{n}} d s=0 .
$$

Além disso, como $u$ é contínua em $\bar{\Omega}$, pelo lema A.2.7

$$
\lim _{r \rightarrow 0^{+}} \int_{\partial B(\xi ; r)} u \frac{\partial F_{\xi}}{\partial \widetilde{n}}=u(\xi),
$$

e, pelo lema A.2.8, como $u \in C^{2}(\bar{\Omega})$, existe o limite

$$
\lim _{r \rightarrow 0^{+}} \int_{\bar{\Omega}_{r}} F_{\xi} \Delta u d \eta=\int_{\bar{\Omega}} F_{\xi} \Delta u d \eta .
$$

$\mathrm{O}$ resultado segue tomando o limite quando $r \rightarrow 0^{+}$em (A-20).

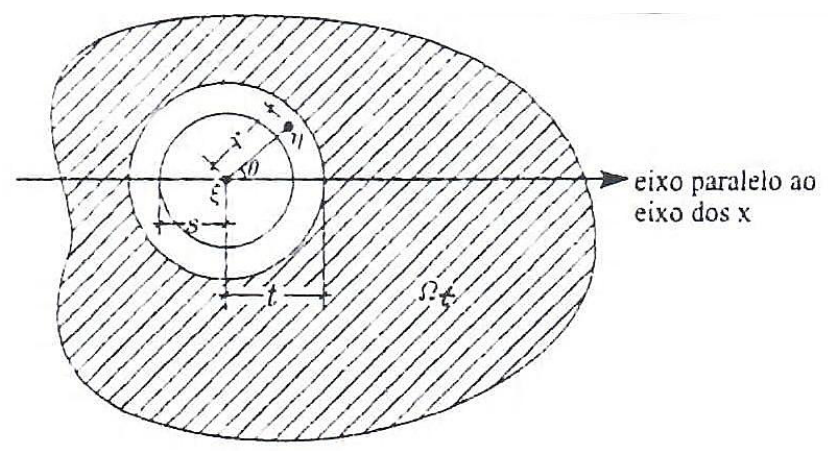

Figura A.4: Sistema de coordenadas polares centrado em $\xi$. 


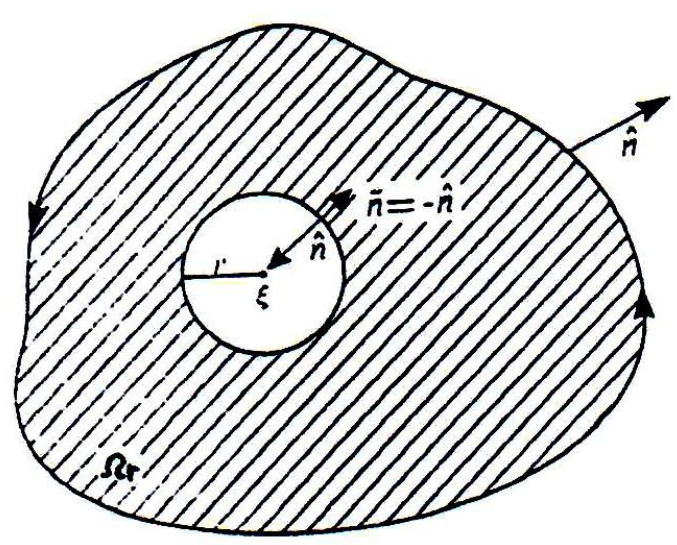

Figura A.5: A orientação positiva de $\partial \Omega_{r}$ coincide com a orientação positiva de $\partial \Omega$ e com a orientação negativa de $\partial B(\xi ; r)$.

\section{A.3}

\section{Identidade de Parseval}

Nesta seção apresentamos um resultado muito útil que relaciona de maneira interessante uma função e sua série de Fourier, e que pode nos auxiliar na estimativa de séries: a identidade de Parseval. Para isto, lembramos que $C_{\text {per }}(2 l)$ denota o espaço das funções $f: \mathbb{R} \rightarrow \mathbb{R}$ contínuas e periódicas de período $2 l$, e $S C_{\text {per }}(2 l)$ o espaço das funções reais periódicas de período $2 l$ que são seccionalmente contínuas em qualquer intervalo $[a, b]$. Lembramos também que nesses espaços podemos introduzir o produto interno $<f, g>=$ $\int_{-l}^{l} f(x) \overline{g(x)} d x$. Vejamos, então, alguns resultados.

Teorema A.3.1 Seja $\left\{f_{n}\right\}_{n \in \mathbb{N}}$ uma sequência em $C_{\mathbb{C}}([a, b])$ e suponha que a sequência $\left\{f_{n}\right\}$ converge uniformemente em $[a, b]$ para uma função $f \in$ $C_{\mathbb{C}}([a, b])$. Então, qualquer que seja $g:[a, b] \rightarrow \mathbb{C}$ seccionalmente contínua,

$$
\int_{a}^{b} f(x) g(x) d x=\lim _{n \rightarrow \infty} \int_{a}^{b} f_{n}(x) g(x) d x .
$$

Prova: Para uma demonstração, veja [19]. No caso mais geral, quando já feito um curso de teoria da medida e integração, pode-se aplicar o teorema da convergência dominada; ver [3] e [18]. 
Teorema A.3.2 Suponha que $f \in C_{\text {per }}(2 l)$ é diferenciável em $(-l, l)$ a menos de um número finito de pontos, com $f^{\prime} \in S C_{\text {per }}(2 l)$. Então a série de Fourier de $f$ converge uniformemente em $\mathbb{R}$ para $f$.

Prova: Para uma demonstração, veja [19].

Teorema A.3.3 Sejam $f, g \in C_{\text {per }}(2 l)$ e suponha que $f$ é diferenciável em $(-l, l)$ a menos de um número finito de pontos, com $f^{\prime} \in S C_{\text {per }}(2 l)$. Então

$$
\frac{1}{2 l}<f, g>=\sum_{n=-\infty}^{\infty} \widehat{f}(n) \overline{\widehat{g}(n)} .
$$

onde $\widehat{f}(n)$ denotam os coeficientes de Fourier complexos da $f$ e analogamente para $\widehat{g}(n)$. Em particular vale a identidade de Parseval

$$
\frac{1}{2 l}\|f\|^{2}=\sum_{n=-\infty}^{\infty}|\widehat{f}(n)|^{2}
$$

Prova: Pelo teorema A.3.2, a série de Fourier de $f$ converge uniformemente para $f$; aplicando então o teorema A.3.1, obtemos:

$$
\begin{aligned}
\frac{1}{2 l}<f, g> & =\frac{1}{2 l} \int_{-l}^{l} f(x) \overline{g(x)} d x \\
& =\frac{1}{2 l} \sum_{n=-\infty}^{+\infty} \widehat{f}(n) \int_{-l}^{l} \overline{g(x)} e^{i n \pi x l} d x \\
& =\sum_{n=-\infty}^{+\infty} \widehat{f}(n) \frac{1}{2 l} \int_{-l}^{l} g(x) e^{-i n \pi x l} d x \\
& =\sum_{n=-\infty}^{+\infty} \widehat{f}(n) \overline{\widehat{g}(n)} .
\end{aligned}
$$

Agora, note que $\frac{1}{2 l}\|f\|^{2}=\frac{1}{2 l}<f, f>=\sum_{n=-\infty}^{+\infty} \widehat{f}(n) \overline{\widehat{f}(n)}=\sum_{n=-\infty}^{\infty}|\widehat{f}(n)|^{2}$.

O teorema A.3.3 é válido em situações bem mais gerais. Por exemplo, as fórmulas em (A-21) e (A-22) continuam válidas se $f, g \in S C_{p e r}(2 l)$, e até para uma classe maior de funções - $f \in L^{2}$ (basta usar argumentos de densidade!).

Na sua forma real, a equação (A-21) torna-se

$$
\frac{1}{l}<f, g>=\frac{a_{0} A_{0}}{2}+\sum_{n=1}^{+\infty}\left(a_{n} A_{n}+b_{n} B_{n}\right)
$$

onde $a_{n}, b_{n}$ e $A_{n}, B_{n}$ são os coeficientes reais de Fourier de $f$ e $g$ respectivamente; assim, a identidade de Parseval pode ser reescrita sob a forma

$$
\frac{1}{l}\|f\|^{2}=\frac{a_{0}^{2}}{2}+\sum_{n=1}^{+\infty}\left(a_{n}^{2}+b_{n}^{2}\right) .
$$




\section{A.4}

\section{Um exemplo de interpolação}

Vamos escrever as condições em $g(r), g^{\prime}(r)$ e $g^{\prime \prime}(r)$ em $r=\frac{1}{2}$ e $r=\frac{1}{2}+\delta$ que torne $\psi$ em (4-8) duas vezes continuamente diferenciável. Neste caso consideramos $\rho=\frac{1}{10}, \delta=\frac{1}{10}$, e encontramos tal $g$ (polinômio de grau 5 ).

Consideremos a função $\psi$ como em (4-8):

$$
\psi(r)= \begin{cases}\frac{1}{2}+\frac{\delta}{1-2 \rho}(r-\rho), & \rho \leq r \leq \frac{1}{2} \\ g(r), & \frac{1}{2}<r<\frac{1}{2}+\delta \\ r, & \frac{1}{2}+\delta \leq r \leq 1\end{cases}
$$

Verifiquemos, então, as condições em $g(r), g^{\prime}(r)$ e $g^{\prime \prime}(r)$ que torne $\psi$ duas vezes continuamente diferenciável. Assim, devemos ter:

1. $g\left(\frac{1}{2}\right)=\frac{1}{2}+\frac{\delta}{1-2 \rho}(r-\rho)$ e $g\left(\frac{1}{2}+\delta\right)=\frac{1}{2}+\delta ;$

2. $g^{\prime}\left(\frac{1}{2}\right)=\frac{\delta}{1-2 \rho}$ e $g^{\prime}\left(\frac{1}{2}+\delta\right)=1$

3. $g^{\prime \prime}\left(\frac{1}{2}\right)=g^{\prime \prime}\left(\frac{1}{2}+\delta\right)=0$.

Por exemplo, podemos tomar $g(r)=\sum_{k=0}^{5} x_{k} r^{k}$ e considerar $\rho=\delta=\frac{1}{10}$. A partir das condições listadas acima, afim de obtermos os coeficientes $x_{k}$ devemos resolver o seguinte sistema linear:

$\begin{cases}0,5^{5} x_{5}+0,5^{4} x_{4}+0,5^{3} x_{3}+0,5^{2} x_{2}+0,5 x_{1}+x_{0} & =0,55 \\ 0,6^{5} x_{5}+0,6^{4} x_{4}+0,6^{3} x_{3}+0,6^{2} x_{2}+0,6 x_{1}+x_{0} & =0,6 \\ 5 \cdot 0,5^{4} x_{5}+4 \cdot 0,5^{3} x_{4}+3 \cdot 0,5^{2} x_{3}+2 \cdot 0,5 x_{2}+0,5 \cdot x_{1}+0 \cdot x_{0} & =0,125 \\ 5 \cdot 0,6^{4} x_{5}+4 \cdot 0,6^{3} x_{4}+3 \cdot 0,6^{2} x_{3}+2 \cdot 0,6 x_{2}+0,6 \cdot x_{1}+0 \cdot x_{0} & =1 \\ 20 \cdot 0,5^{3} x_{5}+12 \cdot 0,5^{2} x_{4}+6 \cdot 0,5 x_{3}+2 \cdot 0,5 x_{2}+0 \cdot x_{1}+0 \cdot x_{0} & =0 \\ 20 \cdot 0,6^{3} x_{5}+12 \cdot 0,6^{2} x_{4}+6 \cdot 0,6 x_{3}+2 \cdot 0,6 x_{2}+0 \cdot x_{1}+0 \cdot x_{0} & =0\end{cases}$ 
Vamos reescrever o sistema na forma $A x=b$, onde

$$
\begin{gathered}
A=\left(\begin{array}{cccccc}
0,03125 & 0,0625 & 0,125 & 0,25 & 0,5 & 1 \\
0,07776 & 0,1296 & 0,216 & 0,36 & 0,6 & 1 \\
0,3125 & 0,500 & 0,75 & 1 & 0,5 & 0 \\
0,6480 & 0,864 & 1.08 & 1,2 & 0,6 & 0 \\
2,5 & 3 & 3 & 1 & 0 & 0 \\
4,32 & 4,32 & 3,6 & 1,2 & 0 & 0
\end{array}\right) \\
\\
x=\left(\begin{array}{c}
x_{5} \\
x_{4} \\
x_{3} \\
x_{2} \\
x_{1} \\
x_{0}
\end{array}\right)
\end{gathered}
$$

e

$$
b=\left(\begin{array}{c}
0,55 \\
0,6 \\
0,125 \\
1 \\
0 \\
0
\end{array}\right)
$$

Com ajuda de um computador, calculamos $\operatorname{det} A=0,001403928>0$ e, portanto, encontraremos $A^{-1}$ para, então, obtermos $x=A^{-1} b$.

$$
A^{-1}=\left(\begin{array}{cccccc}
-46,15621 & 46,15621 & 55,69516 & -54,10534 & -1,412893 & 4,639127 \\
84,61972 & -84,61972 & -102,1078 & 99,19312 & 0,9236371 & -7,116177 \\
-54,10534 & 54,10534 & 58,62044 & -57,86793 & 0,7882199 & 3,401051 \\
23,84738 & -23,84731 & -8,775835 & 11,28776 & -0,6033386 & -0,4524377 \\
-22,30884 & 22,30884 & -1,080671 & -1,150914 & -0,01623160 & 0,02002239 \\
9,109390 & -8,109390 & 0,04800246 & 0,4782297 & 0,04684856 & -0,02224473
\end{array}\right),
$$

$\log \mathrm{O}$

$$
x=\left(\begin{array}{c}
-44,83563261 \\
82,19865976 \\
-47,83510266 \\
8,998410178 \\
-0,1705557525 \\
0,6287604495
\end{array}\right) .
$$


Portanto, $g(r)=-44,83563261 r^{5}+82,19865976 r^{4}-47,83510266 r^{3}+$ $8,998410178 r^{2}-0,1705557525 r+0,6287604495$. 